\title{
TOEPLITZ DETERMINANTS AND SZEGÖ'S FORMULA
}

\author{
R. E. HARTWIG *
}

(Received 22 August 1966; revised 16 August 1967)

\section{Abstract}

In this paper the Toeplitz determinant of order $s \geqq 1$ generated by the rational function

with

$$
f_{m, n}(z)=\frac{u_{n}\left(\frac{1}{z}\right)}{v_{m}(z)}
$$

with

$$
u_{n}\left(\frac{1}{z}\right) \neq 0 \text { for }|z| \geqq r_{1} \text {, }
$$

and

$$
v_{m}(z) \neq 0 \text { for }|z| \leqq r_{2},
$$

is evaluated exactly for all values of $s \geqq m$, as

where

$$
D_{s}\left(f_{m, n}\right)=\exp \left[s k_{0}+\sum_{p=1}^{\infty} p k_{m, p} k_{n,-p}\right]
$$

with

$$
\ln f_{m, n}(z)=\sum_{p=-\infty}^{\infty} k_{p}(m, n) z^{p}
$$

$$
k_{p}(m, n)=\left\{\begin{array}{ll}
k_{m, D} & (p>0) \\
k_{0} & (\phi=0) \\
k_{n,-p} & (\phi<0)
\end{array} \text { and } r_{1}<|z|<r_{2} \quad\left(r_{1} \leqq 1 \leqq r_{2}\right),\right.
$$

thus proving Szego's formula for the function $f_{m, n}(z)$.

By forming the rational approximation of the generating function the formula is then extended to

$$
\lim _{m \rightarrow \infty} D_{m}(f) \cdot e^{-m k_{0}}=\exp \left[\sum_{p=1}^{\infty} p k_{p} k_{-p}\right],
$$

enabling the evaluation of the limit of Toeplitz determinants generated by certain classes of complex valued functions.

\section{Introduction}

In the literature the term Toeplitz determinant [1] is generally used to indicate determinants of the form

* I am grateful to Professor R. B. Potts for initially suggesting this problem, and to Professor G. Szekeres for pointing out some of the literature on resultants. I would also like to thank Professor D. B. Sears for his advice and for many stimulating and informative discussions.

The Commonwealth Post-Graduate Award is gratefully acknowledged by the author. 


$$
\left|c_{p q}\right|=\left|c_{p-q}\right| \text { or }\left|c_{q-p}\right|,
$$

in which the entries depend only upon the difference of their subscripts. Since the two determinants are of equal value we will for convenience assume the former notation.

A Toeplitz determinant is said to be generated by the $L^{\mathbf{1}}$-integrable complex valued function $F(\theta)$ if the entries $c_{p}$ are the Fourier coefficients of $F(\theta)$. It is given and denoted by

$$
D_{s}(F)=\left|c_{p_{q}}\right|_{0}^{s-1}=\left|c_{p-q}\right|_{0}^{s-1}=\left|\frac{1}{2 \pi} \int_{0}^{2 \pi} F(\theta) e^{-i(p-q) \theta} d \theta\right|_{0}^{s-1},
$$

where $s$ is the order ${ }^{1}$ of the determinant and $0 \leqq p, q \leqq s-1$. In general the Fourier coefficients $c_{p}$ are complex numbers and $c_{p} \neq \bar{c}_{-p}$, implying that the associated Toeplitz matrix and form $C_{s}(F)=\left[c_{p-q}\right]$ and $K_{s}=x^{t} C_{2} y$, are non-Hermitian [2]. From the definition (1.2) we note the following important relations for Toeplitz determinants:

$$
\begin{aligned}
\text { (i) } D_{\triangleleft}(1-F) & =\left|I-C_{\triangleleft}(F)\right|, \\
\text { (ii) } D_{\triangleleft}(\alpha \cdot F) & =\alpha^{\varepsilon} \cdot D_{s}(F)
\end{aligned}
$$

(any contant $\alpha$ ),

which follows on taking a factor of $\alpha$ out of each row.

If the function $f(z)$ admits a Laurent expansion $\sum_{p=-\infty}^{\infty} c_{p} z^{p}$ for all $z \in \Omega=\left\{z ; r_{1}<|z|<r_{2}\right\}$, then we can associate with this particular expansion and its corresponding region of convergence the determinant $\left|c_{p-a}\right|$, which when $\Omega$ contains the unit circle $C_{0}$ becomes the Toeplitz determinant $D_{s}(f)$ generated by the function $F(\theta)=f\left(e^{i \theta}\right)$. When $\Omega$ does not contain the unit circle we can still define the determinant $\left|c_{p-a}\right|$, but we will not refer to it as the Toeplitz determinant generated by $f(z)$, but merely as an associated 'improper' Toeplitz determinant of $f(z) .^{2}$ We note that the Toeplitz matrix generated by $f(1 / z)$ is the transpose of the Toeplitz matrix generated by $f(z)$, since both functions have an expansion on $C_{0}$ and $c_{p}$ is interchanged with $c_{-p}$. When the generating function $f(z)$ has real singularities and zeros then the coefficients $c_{p}$ are in general also real and hence $D_{s}(f)$ is real even though $f(z)$ may be complex valued.

In 1952, after hearing about the problem of evaluating the limit of a Toeplitz determinant generated by a function $F,{ }^{3}$ Szegö [3] proved the formula

$$
\lim _{\rightarrow \rightarrow \infty} D_{,}(F) \cdot e^{-s k_{0}}=\exp \left[\sum_{p=1}^{\infty} p\left|k_{p}\right|^{2}\right]
$$

for the limit of Hermitian Toeplitz determinants generated by a certain

1 Often $D_{s}(f)$ denotes $\left|c_{p-\alpha}\right|_{0}^{8}$.

2 Note that it has the same value.

3 See Ref. 14. 
class of real valued positive generating functions $F(\theta)$. In this case use could be made of the theorems on Fourier representation of positive functions, together with the extremal properties of the Hermitian forms and matrices, with $c_{p}=\bar{c}_{-p}$, and their real eigenvalues. ${ }^{4}$

In the literature this formula has extensively been used and quoted for complex valued generating functions, however without satisfactory justification having been given, apart from certain notes and remarks indicating that it could probably be extended. ${ }^{5}$ When the Toeplitz matrix $\left[c_{p-q}\right]$ is non-Hermitian, stronger assumptions have to be made about the generating function before the formula can be applied, in particular when $f(z)$ is not analytic on $C_{0}$, but merely has a Fourier series. The formula essentially aims at expressing the limiting behaviour of a Toeplitz determinant, generated by the Laurent expansion or the Fourier series of $f(z)$, in terms of the singularities and zeros (if any) of the function $f(z)$, which in turn determine the coefficients and the properties of the expansion. We will be mainly concerned with the case where both $f(z)$ and $\ln f(z)$ have a Laurent expansion in $\Omega$ or a Fourier series on $C_{0}$.

\section{An outline of this paper}

The Toeplitz determinant of order $s$ will be investigated on the basis of its eigenvalue equation, which can be written with the aid of the generating function as a homogeneous integral equation. The integral equation will be solved exactly for the product of its eigenvalues using a rational function of the form $f_{m, n}(z)=u_{n}(1 / z) / v_{m}(z)$, with $v_{m}(z) \neq 0$ for $|z| \leqq 1$ and $s \geqq m$, and which serves later as a rational approximation (referred to as rational approximant) of the generating function, which is similar to the Pade approximation.

The Toeplitz determinant $D_{s}\left(f_{m, n}\right)$ will be shown to equal a multiple of a new $(n+s) \times(n+s)$ determinant which for $s \geqq m$ can be identified with the resultant of the polynomials $z^{n} u_{n}(1 / z)$ and $v_{m}(z)$, and hence can be evaluated using the algebraic theory of resultants [4].

The method of finding the product of the eigenvalues of the integral equation is essentially a variation of the Wiener-Hopf technique of separating the kernel, as used by Green [5], when the annulus about infinity, i.e. an infinite strip, is replaced by an annulus about the origin. The results confirm those obtained by Kaufman and Onsager by different means [6].

- It was shortly afterwards shown by Kac, Ref. 15-16, that (1.5) is equivalent to a combinatorial identity in probability theory.

5 We wish to thank Dr. J. Nagle for drawing our attention to the papers by Baxter, Ref. 17-19, who derives the formula using a different approach. For an up-to-date review of the field of finite Toeplitz operators and their application to the Ising model, see Ref. $20-27$. 
From these results Szegö's formula

$$
D_{s}\left(f_{m, n}\right)=\exp \left[s k_{0}+\sum_{p=1}^{\infty} p k_{m, p} k_{n,-p}\right] \quad(s \geqq m)
$$

will be developed, using the residue theorem. This will be extended, on taking the limit as $n \rightarrow \infty$, to

$$
D_{s}\left(t_{m}\right)=\exp \left[s k_{0}+\sum_{p=1}^{\infty} p k_{m, p} k_{-p}\right] \quad(s \geqq m),
$$

where $f_{m}(z)=u(1 / z) / v_{m}(z)$, after which it will be generalized to a larger class of functions by forming this rational approximant of the generating function. Under certain conditions it will be shown that the Toeplitz determinant generated by $f_{m}(z)$ does in fact approximate the Toeplitz determinant generated by the original function $f(z)$, i.e. as $m \rightarrow \infty$

$$
\begin{aligned}
& \text { (i) }\left[D_{m}\left(f_{m}\right)-D_{m}(f)\right] \cdot e^{-m k_{0}} \rightarrow 0 \\
& \text { (ii) } D_{m}\left(f_{m}\right) \cdot e^{-m k_{0}} \rightarrow \exp \left[\sum_{p=1}^{\infty} p k_{p} k_{-p}\right]<\infty .
\end{aligned}
$$

For the former the Landsberg-Pólya representation of a Toeplitz determinant generated by $F(\theta)$, will be used. As a special application of the formula a real valued positive generating function will be considered, verifying the results obtained by Szegö.

\section{The integral equation of a Toeplitz matrix}

The eigenvalue equation of a Toeplitz matrix $\boldsymbol{C}_{s}(f)$ generated by a complex valued function $f(z)$, which admits a Laurent expansion $\sum_{-\infty}^{\infty} c_{p} z^{p}$ for $z \in \Omega=\left\{z ; r_{1}<|z|<r_{2}, r_{1}<1<r_{2}\right\}$, can be written as

$$
c_{s}(f) x=\lambda x
$$

where $\lambda$ is a general eigenvalue and $x^{t}=\left[x_{0}, \cdots, x_{0-1}\right]$ is the corresponding transposed eigenvector. This gives that

$$
\sum_{t=0}^{8-1} c_{r-t} x_{t}=\sum_{t=0}^{8-1} \frac{x_{t}}{2 \pi i} \int_{C} \frac{f(z)}{z^{r-t}} \frac{d z}{z}=\lambda x_{r} \quad(0 \leqq r<s),
$$

since

$$
c_{p}=\frac{1}{2 \pi i} \int_{C} \frac{f(z)}{z^{p+1}} d z
$$

and where $C$ is a contour in $\Omega$ about $O$. And so

$$
\frac{1}{2 \pi i} \int_{C} \frac{f(z)}{z^{r+1}} X_{-1}(z) d z=\lambda x_{r} \quad(0 \leqq r<s),
$$


where $X_{s-1}(z)=x_{0}+x_{1} z+\cdots+x_{s-1} z^{8-1}$. Multiplying this through by $\zeta^{r}$ and summing over $r$ gives

$$
\frac{1}{2 \pi i} \int_{C} f(z) \sum_{r=0}^{s-1}\left(\frac{\zeta}{z}\right)^{r} X_{\sigma-1}(z) \frac{d z}{z}=\lambda X_{\sigma-1}(\zeta)
$$

We note that in this equation we may replace $\lambda$ and $f(z)$ by $1-\lambda$ and $1-f(z)$ respectively. When the contour $C$ is the unit circle this reduces to

$$
\frac{1}{2 \pi} \int_{0}^{2 \pi} f\left(e^{i \theta}\right) \sum_{r=0}^{s-1} e^{i(\phi-\theta) r} X_{\sigma-1}\left(e^{i \theta}\right) d \theta=\lambda X_{s-1}\left(e^{i \phi}\right),
$$

where $\zeta=e^{i \phi}$ and $z=e^{i \theta}$. This integral equation clearly also holds for the eigenvalue equation of the Toeplitz matrix generated by a function $F(\theta)$ which merely has a Fourier series. Hence we have here the general homogeneous integral equation with a kernel $f\left(e^{i \theta}\right) L(\phi, \theta)$, corresponding to the Toeplitz determinant $D_{s}(f)$, and which may be considered as the eigenvalue equation of the projection operator

where

$$
E=\frac{1}{2 \pi} \int_{0}^{2 \pi} d \theta f\left(e^{i \theta}\right) L(\phi, \theta)
$$

$$
L(\phi, \theta)=L(\phi-\theta)=\left(\frac{1-e^{i(\phi-\theta) s}}{1-e^{i(\phi-\theta)}}\right)
$$

A similar result holds for the eigenvalue equation of a general Hankel matrix $\left[c_{p+q}\right]$. The equation (3.5) can unfortunately not be solved in general for the eigenvalues $\lambda_{j}$, however in certain cases, such as with rational functions, the product of the eigenvalues can be found exactly. In a similar way the integral equation of the transposed matrix $C_{s}^{t}(f)$ can be constructed as

$$
\frac{1}{2 \pi i} \int_{C} f\left(\frac{1}{z}\right)^{s-1} \sum_{r=0}\left(\frac{\zeta}{z}\right)^{r} X_{s-1}(z) \frac{d z}{z}=\lambda X_{s-1}(\zeta),
$$

where the $\lambda=\lambda_{j}(1 \leqq j \leqq s)$ are the common eigenvalues of $\boldsymbol{C}_{\mathrm{s}}$ and $\boldsymbol{C}_{\mathrm{s}}^{t}$. The two alternative forms are particularly useful because sometimes the rational approximant $f_{m, n}(z)$ of $f(z)$ does not satisfy certain necessary conditions to ensure that $D_{s}\left(f_{m, n}\right)$ does approximate $D_{s}(f)$, whereas the rational approximant of $f(1 / z)$ may satisfy these conditions, depending on the expansion of $f(z)$.

The structure of the integral equation (3.5) is an inherent property of the Toeplitz determinant. It also appears in the expression for the trace of $\left[C_{s}(f)\right]^{a}$, since 


$$
\begin{aligned}
& T_{r}\left[\boldsymbol{C}_{s}(f)\right]^{q}=\sum_{t_{1}=0}^{s-1} \cdots \sum_{t_{a}=0}^{s-1} c_{t_{1} t_{s}} \cdots c_{t_{q} t_{1}} \\
& =\sum_{t} \frac{1}{(2 \pi)^{q}} \int_{0}^{2 \pi} \cdots \int_{0}^{2 \pi} \exp \left[-i\left[\left(t_{1}-t_{2}\right) \theta_{1}+\cdots+\left(t_{q}-t_{1}\right) \theta_{q}\right]\right] \prod_{r=1}^{q} f\left(e^{i \theta_{r}}\right) d \theta_{r} \\
& =\frac{1}{(2 \pi)^{q}} \int_{0}^{2 \pi} \cdots \int_{0}^{2 \pi} L\left(\theta_{1}-\theta_{2}\right) \cdots L\left(\theta_{q}-\theta_{1}\right) \prod_{r=1}^{q} f\left(e^{i \theta_{r}}\right) d \theta_{r} .
\end{aligned}
$$

This is similar to what (3.5) would give on repeated substitution. The latter however is not a closed summation like (3.8). Combining (1.3) with the fact that for any non-singular square matrix $\boldsymbol{M}$

$$
\ln |\boldsymbol{M}|=\operatorname{Tr} \ln \boldsymbol{M},
$$

yields that

$$
\ln D_{s}(f)=\operatorname{Tr} \ln \left[I-C_{s}(1-f)\right],
$$

which may be expanded, provided that $|1-\lambda|_{\max }<1$, giving with aid of (3.8) that

$$
\begin{aligned}
& \operatorname{In} D_{s}(f)=-\sum_{p=1}^{\infty} \frac{1}{p} \operatorname{Tr}\left[C_{s}(1-f)\right]^{p} \\
& =-\sum_{p=1}^{\infty} \frac{1}{p} \cdot \frac{1}{(2 \pi)^{p}} \int_{0}^{2 \pi} \cdots \int_{0}^{2 \pi} L\left(\theta_{1}-\theta_{2}\right) \cdots L\left(\theta_{e}-\theta_{1}\right) \prod_{r=1}^{p}\left[1-f\left(e^{i \theta_{r}}\right)\right] d \theta_{r},
\end{aligned}
$$

which can be used to investigate the approximations of a Toeplitz determinant. From (3.11) a plausible result similar to that given by Kadanoff [7] can be obtained [8], on breaking the closed summation by introducing a delta function, followed by taking the total derivative.

\section{The solution of the integral equation with a rational function}

Consider the complex rational function

$$
f_{m, n}(z)=\frac{u_{n}\left(\frac{1}{z}\right)}{v_{m}(z)}=\frac{u_{0}+\frac{u_{1}}{z}+\cdots+\frac{u_{n}}{z^{n}}}{v_{0}+v_{1} z+\cdots+v_{m} z^{m}},
$$

with $v_{m}(z) \neq 0$ for $|z| \leqq 1$. Then $1 / v_{m}(z)$ is analytic for $|z| \leqq 1$ and can be expanded as

$$
\frac{1}{v_{m}(z)}=\sum_{p=0}^{\infty} a_{p} z^{p} \quad(|z| \leqq 1),
$$

where 


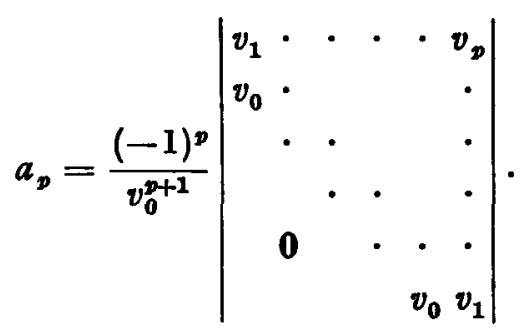

Multiplication by $u_{n}(1 / z)$ shows that $f_{m, n}(z)$ has a Laurent expansion

$$
f_{m, n}(z)=\sum_{p=-\infty}^{\infty} c_{p}(m, n) z^{p} \quad(0<|z| \leqq 1),
$$

with $c_{p}(m, n)=0$ for $p<n$, and which on the unit circle equals the Fourier series of $f_{m, n}\left(e^{i \theta}\right)$.

When $n=0$, it follows that $c_{-p}(m, n)=0$, so that

$$
D_{s}\left(f_{m, 0}\right)=\left(\frac{u_{0}}{v_{0}}\right)^{2} \text {. }
$$

Hence we can assume from now on that $n \geqq 1$.

Consider now the integral equation (3.4) with the function $f(z)$ replaced by the rational function $f_{m, n}(z)$. Then we have that

$$
\frac{1}{2 \pi i} \int_{C} \frac{u_{n}\left(\frac{1}{z}\right)}{v_{m}(z)} \sum_{r=0}^{,-1}\left(\frac{\zeta}{z}\right)^{r} X_{\sigma-1}(z) \frac{d z}{z}=\lambda X_{\sigma-1}(\zeta),
$$

where $C$ is a contour in $\Omega=\{z ; 0<|z| \leqq 1\}$, and which will now be solved exactly for the product of its eigenvalues. To solve (4.4) the ratio $X_{,-1}(z) / v_{m}(z)$ is expanded as a power series

$$
\frac{X_{\sigma-1}(z)}{v_{m}(z)}=y(z)=\sum_{p=0}^{\infty} y_{p} z^{p}
$$

which is permitted by (4.1). On multiplying this by $u_{n}(1 / z)$ and integrating term by term it can be seen that the polynomial $\lambda X_{,-1}(\zeta)$ equals the sum of the first $s$ powers of $z$, taken at $z=\zeta$, in the expansion of

$$
\left(u_{0}+\frac{u_{1}}{z}+\cdots+\frac{u_{n}}{z^{n}}\right)\left(y_{0}+y_{1} z+\cdots+y_{n} z^{n}+\cdots\right) \text {. }
$$

Equating coefficients gives a set of $s$ equations

$$
\sum_{t=0}^{n} u_{t} y_{r+t}=\lambda x_{r} \quad(0 \leqq r<s) .
$$

There are now two cases which have to be considered, viz. when $n+s \geqq m+1$ or $n+s \leqq m+1$. 
CASE (a). $n+s \geqq m+1$.

The set of equations (4.7) may now be written in the augmented matrix form

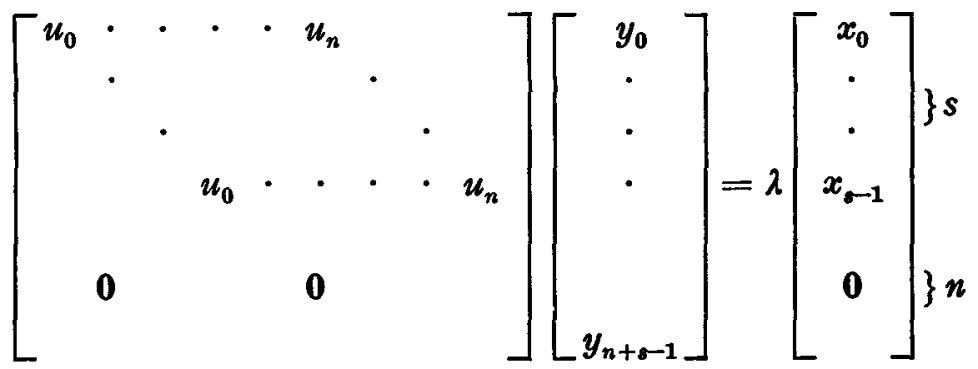

i.e.

$$
\left[\begin{array}{cc}
U_{1} & U_{2} \\
0 & 0
\end{array}\right]\left[\begin{array}{l}
y \\
0
\end{array}\right]=\lambda\left[\begin{array}{l}
x \\
0
\end{array}\right],
$$

where the submatrices $U_{1}$ and $U_{2}$ are of order $s \times s$ and $s \times n$ respectively. The identity $v_{m}(z) \cdot y(z)=X_{s_{-1}}(z)$ gives on equating powers of $z$ another set of $(n+s)$ equations

$$
\sum_{t=0}^{r} v_{r-t} y_{t}= \begin{cases}x_{r} & (0 \leqq r<s) \\ 0 & (s \leqq r<m) \\ x_{r} & (0 \leqq r<m) \quad(m \leqq s)\end{cases}
$$

and

$$
\sum_{t=0}^{m} v_{m-t} y_{r+t}=\left\{\begin{array}{ll}
0 & (0 \leqq r<n+s-m) \\
x_{r+m} & (0 \leqq r<s-m) \\
0 & (s-m \leqq r<n+s-m
\end{array}\right\}_{(m \leqq s)}^{(m \geqq s)}
$$

which in matrix form becomes

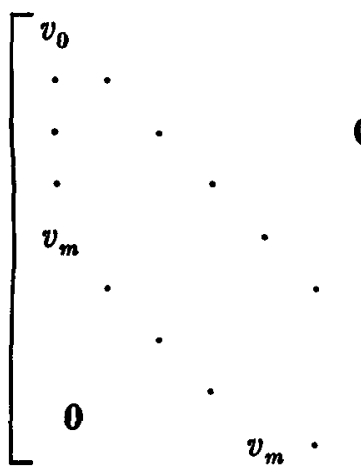

$(4.14)$

$$
\left[\begin{array}{ll}
V_{3} & 0 \\
V_{2} & V_{1}
\end{array}\right]\left[\begin{array}{l}
y
\end{array}\right]=\left[\begin{array}{l}
x \\
0
\end{array}\right],
$$


where the submatrices $V_{1}, V_{2}$ and $V_{3}$ are of order $n \times n, n \times s$ and $s \times s$ respectively, so that their structures depend on the relative values of $m$ and $s$.

Subtracting $\lambda$ times (4.10) and (4.11) from (4.7), combined with (4.12) and (4.13) respectively, gives in matrix form that

$$
\left[\begin{array}{cc}
U_{1}-\lambda V_{3} & U_{2} \\
V_{2} & V_{1}
\end{array}\right][y]=0,
$$

which has a non-trivial solution if and only if

$$
\left|\begin{array}{cc}
U_{1}-\lambda V_{3} & U_{2} \\
V_{2} & V_{1}
\end{array}\right|(n+s) \times(n+s)=0 .
$$

This may be regarded as a polynomial of degree $s$ in $(-\lambda)$ which has exactly the eigenvalues $\lambda_{j}$ of $C_{s}\left(f_{m, n}\right)$ as roots. The coefficient of $\lambda^{s}$ is $\left(-v_{0}\right)^{s} \cdot v_{0}^{n}$ and hence the constant term is $\left(-v_{0}\right)^{s} \cdot v_{0}^{n} \prod_{j=1}^{s}\left(-\lambda_{j}\right)$, giving

$$
v_{0}^{n+s} D_{s}\left(f_{m, n}\right)=\left|\begin{array}{ll}
U_{1} & U_{2} \\
V_{2} & V_{1}
\end{array}\right|
$$

which has the following structure:

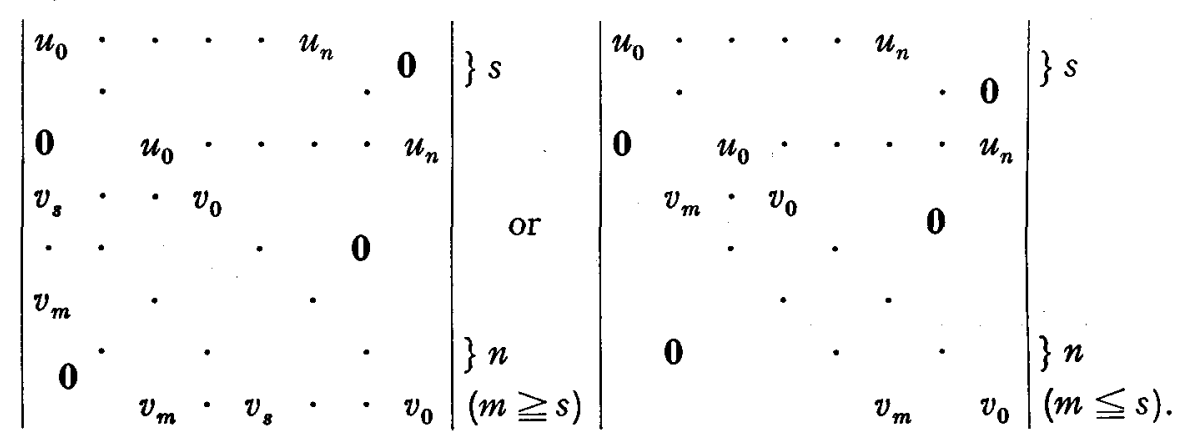

The latter determinant may be expanded by the first $(s-m)$ columns to give for $m \leqq s$ :

$$
\left.u_{0}^{s-m} \cdot\left|\begin{array}{ccccccc}
u_{0} & \cdot & \cdot & \cdot & u_{n} & & \\
& \cdot & & & & & \\
& \cdot & & & & \cdot & \\
& & u_{0} & \cdot & \cdot & \cdot & u_{n} \\
v_{m} & \cdot & \cdot & \cdot & v_{0} & & \\
& \cdot & & & \cdot & \\
& \cdot & & & \cdot & \\
& & v_{m} & \cdot & \cdot & \cdot & v_{0}
\end{array}\right|\right\} n
$$


This expression can be identified exactly with the resultant of the polynomials $[4]$

$$
z^{n} u_{n}\left(\frac{1}{z}\right)=u_{0} z^{n}+\cdots+u_{n}
$$

and

$$
v_{m}(z)=v_{m} z^{m}+\cdots+v_{0},
$$

and will be denoted by $R_{m+n}=R\left[z^{n} u_{n}(1 / z) ; v_{m}(z)\right]$. Hence we obtain for $m \leqq s$ the identity

$$
\begin{aligned}
D_{s}\left(f_{m, n}\right) & =\frac{u_{0}^{s-m}}{v_{0}^{s+n}} R\left[z^{n} u_{n}\left(\frac{1}{z}\right) ; v_{m}(z)\right] \\
& =\left(\frac{u_{0}}{v_{0}}\right)^{s} R\left[z^{n} u_{n}\left(\frac{1}{z}\right) / u_{0} ; v_{m}(z) / v_{0}\right] \quad\left(u_{0} \neq 0 \neq v_{0}\right) .
\end{aligned}
$$

It is clear that $D_{8}$ becomes infinite if $v_{0}=0$, and that it vanishes if $m<s$ and $u_{0}=0$.

It is noteworthy that on repeating this derivation with the normalized rational function

we get that

$$
g_{m, n}(z)=\frac{u_{n}\left(\frac{1}{z}\right) / u_{0}}{v_{m}(z) / v_{0}} \quad\left(u_{0} \neq 0 \neq v_{0}\right),
$$

$$
D_{s}\left(g_{m, n}\right)=R\left[z^{n} u_{n}\left(\frac{1}{z}\right) / u_{0} ; v_{m}(z) / v_{0}\right]
$$

which is what would be expected since (1.4) shows that

$$
D_{s}\left(f_{m, n}\right)=\left(\frac{u_{0}}{v_{0}}\right)^{s} D_{s}\left(g_{m, n}\right)
$$

CASE (b). $n+s \leqq m+1$.

In this case $s \leqq m$ and the set of equations in (4.7) can be expressed in the new $(m+1) \times(m+1)$ augmented matrix form:

$$
\left[\begin{array}{ccc}
U_{1} & U_{2} & \\
& & \\
0 & 0 & \\
& 0 & 0
\end{array}\right]\left[\begin{array}{c}
y_{0} \\
\cdot \\
\cdot \\
y_{m}
\end{array}\right]=\lambda\left[\begin{array}{c}
\boldsymbol{x} \\
\mathbf{0}
\end{array}\right\} s,
$$

where $U_{1}$ and $U_{2}$ are as in (4.9). The identity (4.5) reduces now to 
(4.27)

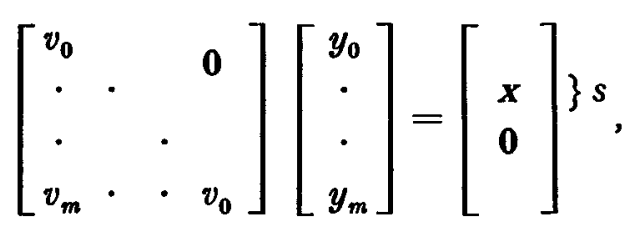

which may be written in the same form as (4.15). After a similar manipulation as in case (a), and on expanding by the last $(m-n-s)$ columns, we arrive at exactly the same expression as in (4.19) with $m \geqq s$, except that this time there are no zero diagonals in the bottom left hand corner, since the coefficients $v_{0}, \cdots, v_{n+\sigma-1}$ are all non-zero. These determinants have the structure of a perturbed resultant and can in general not be evaluated.

In the special case where $m=s$ and $u_{0}=0$, the determinant in (4.20) can be expanded by the $(m+1)^{\text {st }}$ row giving

$$
v_{0}^{m+n} D_{m}\left(f_{m, n}\right)=(-1)^{m} v_{m} R\left[z^{n-1}\left(u_{1}+\frac{u_{2}}{z}+\cdots+\frac{u_{n}}{z^{n-1}}\right) ; v_{m}(z)\right]
$$

And so if we put $u_{r}=-w_{r}(1 \leqq r \leqq n)$, then

$$
D_{m}\left(-\frac{1}{z} \cdot \frac{w_{n-1}\left(\frac{1}{z}\right)}{v_{m}(z)}\right)=\frac{v_{m}}{v_{0}^{m+n}} \cdot R\left[z^{n-1} w_{n-1}\left(\frac{1}{z}\right) ; v_{m}(z)\right] \text {. }
$$

Consider now the case where the generating function has the form

$$
h_{m, n}(z)=z \cdot \frac{u_{n}\left(\frac{1}{z}\right)}{v_{m}(z)},
$$

then we get in exactly similar manner that

$$
\left.v_{0}^{n+8} D_{s}\left(h_{m, n}\right)=\left|\begin{array}{cccccccc}
u_{1} & \cdot & \cdot & \cdot & u_{n} & & & \\
u_{0} & \cdot & & & & \cdot & & 0 \\
\cdot & \cdot & & & & \\
0 & u_{0} & u_{1} & \cdot & \cdot & \cdot & u_{n} & 0 \\
v_{m} & \cdot & \cdot & v_{0} & & & \\
& \cdot & & & \cdot & & 0 \\
& & \cdot & & & \cdot & 0 \\
0 & & \cdot & & & \cdot \\
& & & & v_{m} & \cdot & \cdot & v_{0}
\end{array}\right|\right\} n s
$$

where the structure of the last $n$ rows depends as before on the relative values of $m, n$ and $s$. The only case that is tractable is when $m=n=s$, 
and this gives on expanding by the last column that

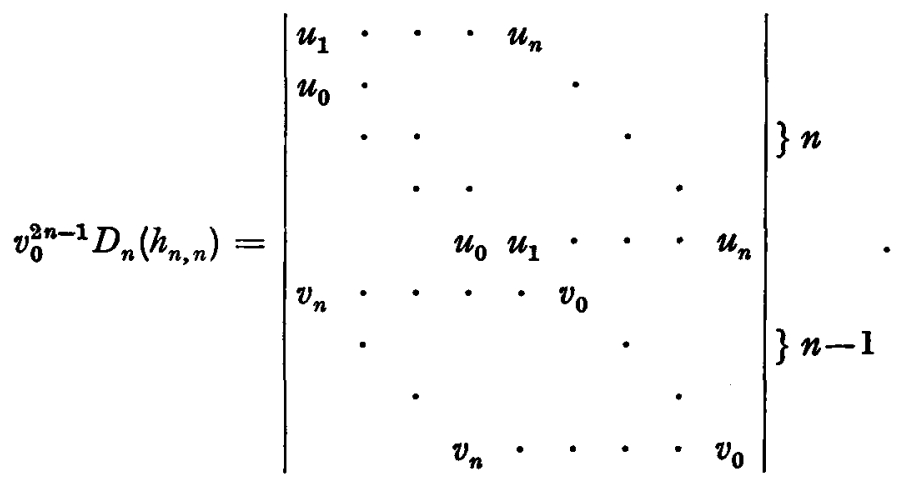

Performing now the row operations:

$$
\text { row }(n-r)-\frac{u_{0}}{v_{n}} \cdot \text { row }(2 n-r-1) \quad(0 \leqq r \leqq n-2),
$$

one obtains

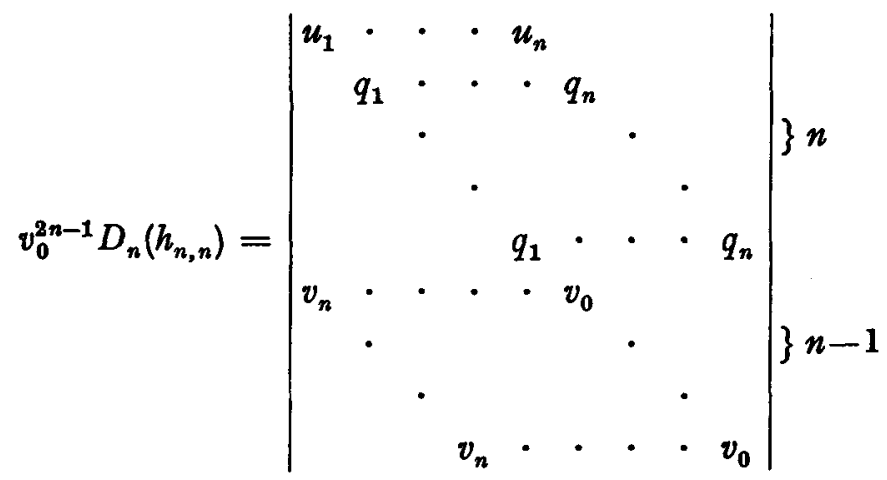

where

$$
q_{r}=u_{r}-\frac{u_{0}}{v_{n}} \cdot v_{n \rightarrow r}
$$

This clearly has the structure of a resultant with a perturbed row and will be investigated as such. If the complete unperturbed matrix is denoted by $M$, and the perturbation matrix by $\eta$, then

where

$$
|M|=R\left[z^{n-1} q_{n-1}\left(\frac{1}{z}\right) ; v_{n}(z)\right] \neq 0,
$$

$$
q_{n-1}(z)=q_{1}+\frac{q_{2}}{z}+\cdots+\frac{q_{n}}{z^{n-1}}=\sum_{r=1}^{n}\left(u_{r}-\frac{u_{0}}{v_{n}} \cdot v_{n-r}\right) z^{1-r}
$$


Whence

$$
\boldsymbol{v}_{0}^{2 n-1} D_{n}\left(h_{n, n}\right)=|\boldsymbol{M}|\left|\boldsymbol{I}+\boldsymbol{\eta} \boldsymbol{M}^{-1}\right|,
$$

where the matrix $\eta$ has all its entries equal to zero, except those in the first row, which equals $\left[\eta_{1}, \cdots, \eta_{n}, 0, \cdots, 0\right]$ with

$$
\eta_{r}=\frac{u_{0}}{v_{n}} \cdot v_{n-r}
$$

If $\boldsymbol{M}^{-1}=\boldsymbol{B}=\left[b_{i j}\right]$, then

$$
\left|\boldsymbol{I}+\boldsymbol{\eta} \boldsymbol{M}^{-1}\right|=\mathbf{1}+\frac{u_{0}}{v_{n}} \cdot \sum_{r=1}^{n} b_{r, 1} v_{n-r},
$$

which when substituted in (4.35) gives an expression for $D_{n}$. We note that in the case where $m=n=s+1$, equation (4.19) yrelds a similar structure to that exhibited in (4.31), except that this time the last $n$ rows have an additional non-zero diagonal.

Before we can proceed with the evaluation of these expressions we have to find the first column in the inverse $B$, which will be done in the next section.

\section{The resultant}

The resultant of two polynomials is a rational integral form in the coefficients of the two polynomials, which vanishes if the two polynomials have a non-constant common factor, or if the leading coefficient vanishes in both of them, and conversely. It is closely related to Euclid's algorithm and occurs frequently in the theory of polynomials, rational functions and continued fractions [9] [10]. Its usefulness lies in the fact that it can be expressed in terms of the zeros of the polynomials which generate it.

The resultant of the two polynomials

$$
p_{n}(z)=a_{0} \prod_{j=1}^{n}\left(z-p_{j}\right) \quad \text { and } \quad q_{m}(z)=b_{0} \prod_{k=1}^{m}\left(z-q_{k}\right)
$$

with $a_{0} \neq 0 \neq b_{0}$, is obtained by considering the equation

$$
p_{n}(z) h_{m-1}(z)=q_{m}(z) k_{n-1}(z),
$$

which on equating powers of $\boldsymbol{z}$ gives the set of $(m+n)$ equations $\boldsymbol{M}^{t} \boldsymbol{y}=\mathbf{0}$, in the unknown coefficients of $h_{m-1}(z)$ and $-k_{n-1}(z)$, where

and

$$
\boldsymbol{y}^{t}=\left[h_{0}, \cdots, h_{m-1},-k_{0}, \cdots,-k_{n-1}\right] \text {, }
$$




$$
\left.|\boldsymbol{M}|=\left|\begin{array}{cccccccc}
a_{0} & \cdot & \cdot & \cdot & a_{n} & & & \\
& \cdot & & & & \cdot & & \\
& & \cdot & & & & \cdot & \\
& & & a_{0} & \cdot & \cdots & \cdot & a_{n} \\
b_{0} & \cdot & \cdot & \cdot & b_{m} & & \\
& \cdot & & & & \cdot & \\
& & \cdot & & & & \cdot \\
& & b_{0} & \cdot & \cdot & \cdot & b_{m}
\end{array}\right|\right\} n
$$

This set of equations will have a non-trivial solution if and only if the determinant vanishes implying after factorization into prime factors, that $p_{n}(z)$ and $q_{m}(z)$ must have a common factor. This determinant is the usual way of representing the resultant, and is clearly homogeneous of degree $m$ in $a_{i}$ and homogeneous of degree $n$ in $b_{j}$, and has a leading term of $a_{0}^{m} b_{m}^{n}$.

It can be shown that the resultant may be expressed in terms of the zeros of the polynomials concerned [4]. In fact

$$
\begin{aligned}
R\left[p_{n}(z) ; q_{m}(z)\right] & =a_{0}^{m} b_{0}^{n} \prod_{j=1}^{n} \prod_{k=1}^{m}\left(p_{j}-q_{k}\right) \\
& =a_{0}^{m} \prod_{j=1}^{n} q_{m}\left(p_{j}\right)=(-1)^{m n} b_{0}^{n} \prod_{k=1}^{m} p_{n}\left(q_{k}\right) .
\end{aligned}
$$

Furthermore we have that:

$$
R\left[p_{n}(z) ; q_{m}(z)\right]=R\left[q_{m}(z) ; p_{n}(z)\right],
$$

which can be verified directly on forming $\boldsymbol{J} \boldsymbol{M} \boldsymbol{J}$, where

$$
\boldsymbol{J}=\left[\delta_{i+j, m+n+1}\right] \quad(1 \leqq i, j \leqq m+n) .
$$

In particular if the polynomials

and

$$
p_{n}(z)=z^{n} u_{n}\left(\frac{1}{z}\right)=u_{0} z^{n}+u_{1} z^{n-1}+\cdots+u_{n}=u_{0} \prod_{j=1}^{n}\left(z-\alpha_{j}\right)
$$

$$
q_{m}(z)=v_{m}(z)=v_{m} z^{m}+\cdots+v_{1} z+v_{0}=v_{m} \prod_{k=1}^{m}\left(z-\beta_{k}\right)
$$

are considered then

$$
R_{m+n}=R\left[z^{n} u_{n}\left(\frac{1}{z}\right) ; v_{m}(z)\right]=u_{0}^{m} \prod_{j=1}^{n} v_{m}\left(\alpha_{j}\right)=v_{0}^{n} \prod_{k=1}^{m} u_{n}\left(\frac{1}{\beta_{k}}\right),
$$

where use was made of the fact that

$$
v_{m} \prod_{k=1}^{m}\left(-\beta_{k}\right)=v_{0} .
$$


An alternative expression for this resultant, which is rather useful, is that

$$
R_{m+n}=\prod_{j=1}^{m+n} v_{m}\left(\rho_{j}\right)=\prod_{j=1}^{m+n} u_{n}\left(\frac{1}{\rho_{j}}\right)
$$

where the $\rho_{j}(1 \leqq j \leqq m+n)$, are the $(m+n)$ roots of the equation

$$
u_{n}\left(\frac{1}{z}\right)=v_{m}(z)
$$

This can be shown either by using the determinantal expression for $R_{m+n}$ or by analytical means. The most direct way however is to make use of the eigenvalue equation and to substitute a trial eigenvector whose transpose has the form:

$$
x^{t}=\left[1, \rho^{-1}, \rho^{-2}, \cdots, \rho^{1-m-n}\right] .
$$

This gives that $\boldsymbol{M x}=\lambda \boldsymbol{x}$ becomes

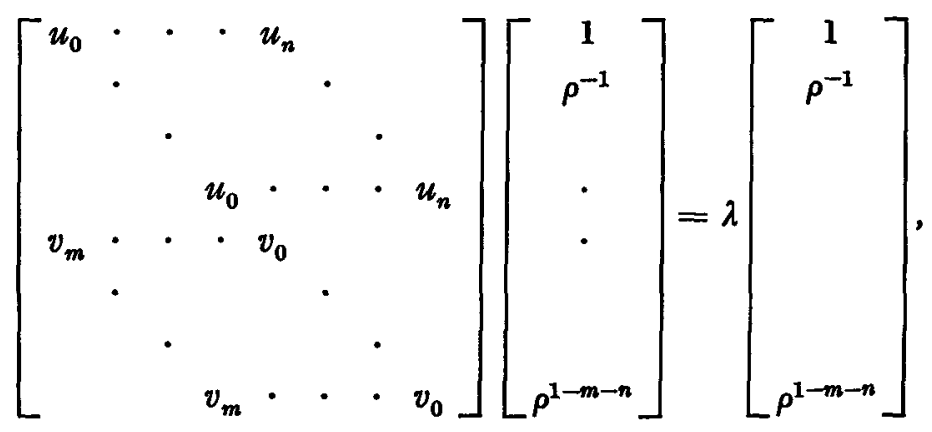

in which the first $m$ equations may be written as

$$
\rho^{-r}\left[u_{0}+u_{1} \rho^{-1}+\cdots+u_{n} \rho^{-n}\right]=\lambda \rho^{-r}
$$$$
(0 \leqq r<m)
$$

and the last $n$ equations become

$$
\rho^{-m-r}\left[v_{m} \rho^{m}+\cdots+v_{1} \rho+v_{0}\right]=\lambda \rho^{-m-r} \quad(0 \leqq r<n) .
$$

These equations are identically satisfied if and only if

$$
\lambda_{j}=u_{n}\left(\frac{1}{\rho_{j}}\right)=v_{m}\left(\rho_{j}\right) \quad(1 \leqq j \leqq m+n),
$$

from which the identity (5.7) follows. It is clear that the eigenvalues and eigenvectors of the matrix $M$ in (5.10) are given by (5.13) and (5.9) respectively. It follows immediately that $\boldsymbol{M}$ can be diagonalized by the similarity transformation $\boldsymbol{P}^{-1} \boldsymbol{M P}$, where

$$
P=\left[x_{1}, \cdots, x_{m+n}\right]
$$


and $x_{j}$ is the eigenvector corresponding to $\lambda_{j}$, as can be seen from

$$
\boldsymbol{M}\left[\boldsymbol{x}_{1}, \cdots, \boldsymbol{x}_{m+n}\right]=\left[\boldsymbol{x}_{1}, \cdots, \boldsymbol{x}_{m+n}\right]\left[\lambda_{1} \dot{+} \cdots \dot{+} \lambda_{m+n}\right] .
$$

Hence

$$
\boldsymbol{M}^{-1}=\left[\boldsymbol{x}_{1} / \lambda_{1}, \cdots, \boldsymbol{x}_{m+n} / \lambda_{m+n}\right] \boldsymbol{P}^{-1} .
$$

The matrix $\boldsymbol{P}$ is now a simple Van der Monde matrix whose inverse $\boldsymbol{A}$ can be calculated since the $\rho_{j}$ are distinct in general. Especially the first column of $\boldsymbol{A}$, which is useful in perturbation theory, can simply be evaluated ${ }^{\circ}$.

From the determinantal representation (5.2) it is clear that if $m \leqq n$, we may subtract the last $m$ rows from the first $m$ rows and conversely if $m \geqq n$ we may subtract the first $n$ rows from the last $n$ rows, showing that

$$
R\left[p_{n}(z) ; q_{m}(z)\right]= \begin{cases}R\left[p_{n}(z)-q_{m}(z) ; q_{m}(z)\right] & (m \leqq n) \\ R\left[p_{n}(z) ; q_{m}(z)-p_{n}(z)\right] & (m \geqq n) .\end{cases}
$$

This simply restates the fact that if $p_{r}(z)$ and $q_{m}(z)$ have a common zero, then so do $\pm\left[p_{n}(z)-q_{m}(z)\right], p_{n}(z)$ and $q_{m}(z)$. When $m=n$ we get equality in both parts of (5.17). If now the resultant is considered of the polynomials

$$
\text { and } \quad p_{n}(z)=z^{n} u_{n}\left(\frac{1}{z}\right)=u_{0} z^{n}+\cdots+u_{n}
$$

then

$$
q_{m+n}(z)=z^{n} v_{m}(z)=v_{m} z^{m+n}+\cdots+v_{0} z^{n},
$$

$$
\left.R\left[z^{n} u_{n}\left(\frac{1}{z}\right) ; z^{n} v_{m}(z)\right]=\left|\begin{array}{rrrrrrr}
u_{0} & \cdot & \cdot & \cdot & u_{n} & & \\
& \cdot & & & & \cdot & \\
& \cdot & & & \cdot & 0 \\
0 & & \cdot & & & \cdot \\
& & & u_{0} & \cdot & \cdot & \cdot \\
v_{m} & \cdot & \cdot & v_{0} & & & \\
& \cdot & & & \cdot & & \\
& \cdot & & \cdot & 0 \\
0 & & v_{m} & \cdot & \cdot & v_{0}
\end{array}\right|\right\} m+n
$$

which on expanding by the last $n$ columns gives

$$
\left(u_{n}\right)^{n}(-1)^{n^{2}} R\left[z^{n} u_{n}\left(\frac{1}{z}\right) ; v_{m}(z)\right] \text {. }
$$

However on subtracting the second-last $n$ rows from the last $n$ rows it can also be seen to equal

- For non-simple Van der Monde determinants see Ref. 10, Sec. 337. 


$$
R\left[z^{n} u_{n}\left(\frac{1}{z}\right) ; z^{n}\left[v_{m}(z)-u_{n}\left(\frac{1}{z}\right)\right]\right]
$$

Expressing this now in terms of the zeros of the polynomials using (5.4), and equating it to (5.20) gives that

$$
R\left[z^{n} u_{n}\left(\frac{1}{z}\right) ; v_{m}(z)\right]=(-1)^{(m+n) n-n^{2}}\left(\frac{v_{m}}{u_{n}}\right)^{n} \prod_{j=1}^{m+n}\left(\rho_{j}\right)^{n} u_{n}\left(\frac{1}{\rho_{j}}\right) .
$$

Since

$$
z^{n}\left[v_{m}(z)-u_{n}\left(\frac{1}{z}\right)\right]=v_{m} z^{m+n}+\cdots-u_{n},
$$

it is clear that

$$
\prod_{j=1}^{m+n} \rho_{j}=-(-1)^{m+n} \frac{u_{n}}{v_{m}}
$$

which when substituted in (5.21) gives the identity between (5.6) and (5.7), and where the $\rho_{j}$ are again the roots of (5.8). It is noteworthy that the identity may also be proved using complex variable theory, such as the residue theorem and a contour $C$ which separates the zeros of $u_{n}(1 / z)$ and $v_{m}(z)$. A modified version of the maximum - minimum modulus theorem applied to an annulus about 0 is also needed.

From (5.13) it follows now that the eigenvalues of the matrix $\boldsymbol{M}$ defined as in (4.35) are

$$
\lambda_{j}=q_{n-1}\left(\frac{1}{\rho_{j}}\right)=v_{n}\left(\rho_{j}\right) \quad(1 \leqq j<2 n),
$$

and that the corresponding transposed eigenvectors are

$$
x_{j}^{t}=\left[1, \rho_{j}^{-1}, \cdots, \rho^{2-2 n}\right] .
$$

It is clear from (4.36) that only the first column in the inverse matrix $\boldsymbol{M}^{-1}$ is wanted, and so by (5.16) only the first column of $\boldsymbol{A}$ is needed. We thus obtain that

$$
b_{r, 1}=\sum_{j=1}^{2 n-1} \frac{\left(\rho_{j}\right)^{1-r}}{\lambda_{j}} a_{j, 1}
$$

Substituting this in (4.36) yields after interchanging the summations and using the definition of $v_{n}(z)$, that

$$
v_{0}^{2 n-1} D_{n}\left(h_{n, n}\right)=|M|\left[1+\frac{1}{v_{n}} \sum_{j=1}^{2 n-1} a_{j, 1}\left(\rho_{j}\right)^{1-n}-\sum_{j=1}^{2 n-1} \frac{a_{j, 1} \rho_{j}}{v_{n}\left(\rho_{j}\right)}\right] .
$$

The resultant $|\boldsymbol{M}|$ can now be evaluated by (5.6) as 


$$
q_{1}^{n} \prod_{j=1}^{n-1} v_{n}\left(\alpha_{j}\right)=v_{n}^{n-1} \prod_{k=1}^{n} q_{n-1}\left(\frac{1}{\beta_{k}}\right)
$$

whereas the first sum vanishes exactly by the relation

$$
\boldsymbol{A} \boldsymbol{P}=\boldsymbol{I}=\boldsymbol{P} \boldsymbol{A} \quad \text { or } \quad \sum_{j=1}^{2 n-1}\left(\rho_{j}\right)^{1-m} a_{j, 1}=\delta_{m, 1} \quad(1 \leqq m<2 n) .
$$

The second term however, does not permit a simpler exact evaluation even though the entries $a_{i, 1}$ can be computed exactly, since the cofactors are again Van der Monde determinants.

\section{The contour integral and Szegö's formula}

THEOREM 1. If

$$
f_{m, n}(z)=\frac{u_{n}\left(\frac{1}{z}\right)}{v_{m}(z)}=\frac{u_{0}+\frac{u_{1}}{z}+\cdots+\frac{u_{n}}{z^{n}}}{v_{0}+v_{1} z+\cdots+v_{m} z^{m}},
$$

where $u_{n}(1 / z)$ and $v_{m}(z)$ are non-zero for $|z| \geqq r_{1}$ and $|z| \leqq r_{2}$ respectively, and where $r_{1}<1<r_{2}$ and $u_{0} \neq 0 \neq v_{0}$, and if $\ln f_{m, n}(z)=\sum_{p=-\infty}^{\infty} k_{p}(m, n) z^{p}$ for $z \in \Omega=\left\{z ; r_{1}<|z|<r_{2}\right\}$, with

and $s \geqq m$, then

$$
k_{p}(m, n)= \begin{cases}k_{m, p} & (p>0) \\ k_{0} & (p=0) \\ k_{n,-p} & (p<0)\end{cases}
$$

$$
D_{s}\left(f_{m, n}\right)=\exp \left[s k_{0}+\sum_{p=1}^{\infty} p k_{m, p} k_{n,-p}\right] .
$$

Proof. It is clear that $f_{m, n}(z)$ is analytic for $0<|z| \leqq \boldsymbol{r}_{2}$ and hence its Laurent expansion on $C_{0}$ coincides with the Fourier series of $f_{m, n}\left(e^{i \theta}\right)$. From equations (4.22) and (5.6) it follows that

$$
\begin{aligned}
\left(\frac{v_{0}}{u_{0}}\right)^{s} D_{s}\left(f_{m, n}\right) & =\prod_{j=1}^{n} v_{m}\left(\alpha_{j}\right) / v_{0}=\prod_{k=1}^{m} u_{n}\left(\frac{1}{\beta_{k}}\right) / u_{0} \\
& =\exp \left[\sum_{j=1}^{n} \ln \left(v_{m}\left(\alpha_{j}\right) / v_{0}\right)\right],
\end{aligned}
$$

where $u_{n}\left(1 / \alpha_{j}\right)=v_{m}\left(\beta_{k}\right)=0$. Since $u_{n}(1 / z)$ and $v_{m}(z)$ are polynomials which are non-zero for $|z| \geqq r_{1}$ and $|z| \leqq r_{2}$ respectively, it can be deduced that the principal branches of $\ln u_{n}(1 / z)$ and $\ln v_{m}(z)$ are analytic in the corresponding regions and may be expanded as 
giving and

$$
\ln \left(u_{n}\left(\frac{1}{z}\right) / u_{0}\right)=\sum_{p=1}^{\infty} k_{n,-p} z^{-p}
$$

$$
\ln \left(v_{m}(z) / v_{0}\right)=-\sum_{p=1}^{\infty} k_{m, p} z^{p}
$$

where

and

$$
\ln f_{m, n}(z)=\sum_{p=-\infty}^{\infty} k_{p}(m, n) z^{p}
$$

$$
k_{p}(m, n)= \begin{cases}k_{m, D} & (p>0) \\ k_{0} & (p=0) \\ k_{n,-p} & (p<0)\end{cases}
$$

$$
\frac{u_{0}}{v_{0}}=e^{k_{0}}=\frac{1}{2 \pi} \int_{0}^{2 \pi} \ln f_{m, n}\left(e^{i \theta}\right) d \theta .
$$

This constant factor is often referred to as the geometric mean $G\left(f_{m, n}\right)$ of $f_{m, n}\left(e^{i \theta}\right)$. We can now apply the residue theorem to (6.2), which yields:

$$
D_{s}\left(f_{m, n}\right)=\exp \left[s k_{0}+\frac{1}{2 \pi i} \int_{C} \ln \left(v_{m}(z) / v_{0}\right) \frac{d}{d z} \ln u_{n}\left(\frac{1}{z}\right) d z\right] .
$$

where $C$ is any contour in $\Omega$ about 0 . Alternatively this may be written as

$$
\exp \left[s k_{0}+\frac{1}{2 \pi i} \int_{-C} \ln \left(u_{n}\left(\frac{1}{z}\right) / u_{0}\right) \frac{d}{d z} \ln v_{m}(z) d z\right] .
$$

Substituting the expansions of (6.3) gives

$$
\begin{aligned}
D_{s}\left(f_{m, n}\right) & =\exp \left[s k_{0}+\frac{1}{2 \pi i} \int_{C} \sum_{p, q=1}^{\infty} q k_{m, p} k_{n,-q} z^{p-q-1} d z\right] \\
& =\exp \left[s k_{0}+\sum_{p=1}^{\infty} p k_{m, p} k_{n,-p}\right],
\end{aligned}
$$

which is exact for all finite $m, n$ and $s$ with $s \geqq m$. Termwise integration is permitted since $r_{1}<1<r_{2}$, which causes the convergence to be uniform on $\Omega$ and hence on the contour $C$. From (6.8) we can deduce for $s \geqq m$ the following product rule:

$$
\begin{aligned}
& D_{2 a}\left(\frac{u_{n}\left(\frac{1}{z}\right)}{v_{m}(z)} \cdot \frac{p_{n}\left(\frac{1}{z}\right)}{q_{m}(z)}\right) \\
= & D_{*}\left(\frac{u_{n}\left(\frac{1}{z}\right)}{v_{m}(z)}\right) \cdot D_{*}\left(\frac{p_{n}\left(\frac{1}{z}\right)}{q_{m}(z)}\right) \cdot D_{*}\left(\frac{u_{n}\left(\frac{1}{z}\right)}{q_{m}(z)}\right) \cdot D_{\cdot}\left(\frac{p_{n}\left(\frac{1}{z}\right)}{v_{m}(z)}\right),
\end{aligned}
$$


for the polynomials $u_{n}, v_{m}, p_{n}$ and $q_{m}$ which satisfy the conditions of theorem 1 . We further note that if, as $n \rightarrow \infty$,

$$
\begin{aligned}
& \text { (1) } u_{n}\left(\frac{1}{z}\right) \rightarrow u\left(\frac{1}{z}\right) \text { dominatedly for }|z|>r_{1} \quad\left(r_{1} \leqq 1\right) \\
& \begin{array}{ll}
\text { (2) } k_{n,-p} \rightarrow k_{-p} \\
\text { (3) } \sum_{p=1}^{\infty} p\left|k_{m, p}\right| \quad \text { converges for all } m,
\end{array}
\end{aligned}
$$

then we may take the limit as $n \rightarrow \infty$ on both sides of the equation in (6.8) giving

$$
D_{s}\left(\frac{u\left(\frac{1}{z}\right)}{v_{m}(z)}\right)=\exp \left[s k_{0}+\sum_{p=1}^{\infty} p k_{m, p} k_{-p}\right] .
$$

Corollary. The theorem can be extended to the case where $u_{n}\left(e^{-i \theta}\right) \neq 0$ and $v_{m}(z) \neq 0$ for $|z| \leqq 1$.

Proof. Since $u_{n}\left(e^{-i \theta}\right)$ and $v_{m}\left(e^{i \theta}\right)$ are non-zero polynomials which are continuous and bounded and $1 / v_{m}(z)$ is analytic for $|z| \leqq 1$, it follows that

and

$$
\ln \left(v_{m}\left(e^{i \theta}\right) / v_{0}\right)=-\sum_{p=1}^{\infty} k_{m, p} e^{i p \theta}
$$

$$
\frac{u_{n}^{\prime}\left(e^{-i \theta}\right)}{u_{n}\left(e^{-i \theta}\right)}\left(-i e^{-i \theta}\right)=-\sum_{q=-\infty}^{\infty} i q l_{n,-q} e^{-i q \theta} .
$$

Both functions are in $L^{2}(0,2 \pi)$ and hence by Parseval's theorem the product of their (uniformly convergent) Fourier series may be integrated term by term giving the required result.

Consider now as a special application equation (4.29) and suppose that the functions $w_{n-1}(1 / z)$ and $v_{m}(z)$ satisfy the conditions of theorem 1 . Then we obtain from (5.6), (6.8) and (6.0), on replacing $u_{n}(1 / z)$ by $w_{n-1}(1 / z)$, that

$$
\begin{aligned}
D_{m}(- & \left.\frac{1}{z} \cdot \frac{w_{n-1}\left(\frac{1}{z}\right)}{v_{m}(z)}\right)=\frac{v_{m}}{v_{0}}\left(\frac{w_{0}}{v_{0}}\right)^{m} \prod_{j=1}^{n-1} v_{m}\left(\alpha_{j}\right) \\
& =\frac{v_{m}}{v_{0}} \cdot \exp \left[m k_{0}+\sum_{p=1}^{\infty} p k_{m, p} k_{n-1,-p}\right]=\frac{v_{m}}{v_{0}} D_{m}\left(\frac{w_{n-1}\left(\frac{1}{z}\right)}{v_{m}(z)}\right),
\end{aligned}
$$

where $w_{n-1}\left(1 / \alpha_{j}\right)=0$. If $(6.10)$ is satisfied we may again take the limit as $n$ tends to infinity on both sides of the equation. 


\section{The Laurent expansion}

Theorem 1 can now be used to extend the formula to generating functions which admit a Laurent expansion.

Theorem 2. If the function $f(z)$ admits a Laurent expansion $\sum_{p--\infty}^{\infty} c_{p} z^{p}$ which is convergent and non-zero for $z \in \Omega=\left\{z ; r_{1}<|z|<r_{2}, r_{1}<1<r_{2}\right\}$, and if $\ln f(z)=\sum_{-\infty}^{\infty} k_{p} z^{p}$ for $z \in \Omega$ and $\left|f\left(e^{i \theta}\right)\right| \leqq r_{0}=\min \left(1 / r_{1}, r_{2}\right)$, then

$$
\lim _{m \rightarrow \infty} D_{m}(f) e^{-m k_{0}}=\exp \left[\sum_{p=1}^{\infty} p k_{p} k_{-p}\right] \text {. }
$$

Proof. Since $f(z)$ is analytic and non-zero for $z \in \Omega$ it follows that the principal branch of $\ln f(z)$ is analytic in the same region and can be expanded in a Laurent series $\sum_{-\infty}^{\infty} k_{p} z^{p}$. Hence $\sum_{p=1}^{\infty} k_{-p} z^{-p}$ and $\sum_{p=1}^{\infty} k_{p} z^{p}$ are analytic functions for $|z|>r_{1}$ and $|z|<r_{2}$ respectively. The function $f(z)$ can now be written in the form

$$
f(z)=\frac{u\left(\frac{1}{z}\right)}{v(z)}=\frac{u_{0}}{v_{0}} \cdot\left[\frac{u\left(\frac{1}{z}\right) / u_{0}}{v(z) / v_{0}}\right]
$$

by defining and

$$
u\left(\frac{1}{z}\right) / u_{0}=\exp \left[\sum_{p=1}^{\infty} k_{-p} z^{-p}\right]
$$

$$
v(z) / v_{0}=\exp \left[-\sum_{p=1}^{\infty} k_{p} z^{p}\right]
$$

together with

$$
\frac{u_{0}}{v_{0}}=e^{k_{0}} \quad\left(u_{0} \neq 0 \neq v_{0}\right),
$$

from which it is clear that $u(1 / z)$ and $v(z)$ are analytic for $|z|>r_{1}$ and $|z|<r_{2}$ respectively. It is sometimes more convenient to take the form of $f(z)$ in (7.1), rather than the existence of the Laurent expansion of $\ln f(z)$, as the starting point of the investigation. Expanding the series in their respective regions of convergence gives

$$
\begin{aligned}
u\left(\frac{1}{z}\right) & =u_{0}+\frac{u_{1}}{z}+\cdots+\frac{u_{n}}{z^{n}}+\cdots & & \left(|z|>r_{1}\right) \\
v(z) & =v_{0}+v_{1} z+\cdots+v_{m} z^{m}+\cdots & & \left(|z|<r_{2}\right) .
\end{aligned}
$$

From (7.1) and (1.4) it is clear that without loss of generality we may assume that $u_{0}=v_{0}=1$. For sufficiently large $m$ and $n$, i.e. for all $m, n>N_{0}$, the partial sums $u_{n}(1 / z)$ and $v_{m}(z)$ will, by Hurwitz's theorem 
[12], be non-zero for $|z|>r_{1}$ and $|z|<r_{2}$ respectively, and hence on forming the rational approximant

$$
f_{m, n}(z)=\frac{u_{n}\left(\frac{1}{z}\right)}{v_{n}(n)},
$$

theorem $\mathrm{I}$ can be applied to give for $s \geqq m$

$$
D_{a}\left(f_{m, n}\right)=D_{m}\left(f_{m, n}\right)=\exp \left[\sum_{p=1}^{\infty} p k_{m, p} k_{n,-p}\right] \text {. }
$$

The condition that $f(z)$ and $\ln f(z)$ are analytic inside $\Omega$ is a very strong assumption which ensures that all the convergence that will be dealt with in this section is uniform and in fact exponentially fast. It should be noted that only the singularities and/or zeros of $f(z)$ on the boundaries of $\Omega$ do determine the behaviour of $D_{m}(f)$, in particular if $u(1 / z)$ and $v(z)$ have a common zero on $C_{0}$, then $D_{m}(f)$ vanishes as $m \rightarrow \infty$.

The conditions of (6.10) are clearly satisfied since $u(1 / z)$ is analytic for $|z|>r_{1}$ and the coefficients $k_{m, p}$ and $k_{p}$ are the Laurent coefficients of functions analytic inside $\Omega$ with $r_{1}<1<r_{2}$ and $r_{0}>1$. We have in fact that

$$
\begin{array}{ll}
\text { (i) }\left|k_{p}\right|=O\left(\frac{1}{r_{0}^{p}}\right),\left|k_{m, p}\right|=O\left(\frac{1}{r_{0}^{p}}\right), & \\
\text { (ii) } k_{m, p}=k_{p} & (p \leqq m), \\
\text { (iii) }\left|k_{m, p}-k_{p}\right|=O\left(\frac{1}{r_{0}^{p}}\right) & (p>m) .
\end{array}
$$

The second relation is a direct consequence of the definition

$$
\ln v_{m}(z)=\sum_{q=1}^{\infty} \frac{(-1)^{q}}{q}\left(v_{1} z+\cdots+v_{m} z^{m}\right)^{q}=-\sum_{p=1}^{\infty} k_{m, p} z^{p},
$$

and the third relation follows from the fact that

$$
k_{m, p}-k_{p}=\frac{1}{2 \pi i} \int_{C} \sum_{q=1}^{\infty} \frac{1}{q}\left[\frac{Q_{m+1}(z)}{v(z)}\right]^{q} \frac{d z}{z^{p+1}},
$$

where $Q_{m+1}(z)=v(z)-v_{m}(z)$ and $C$ is in $\Omega$. After interchanging the summation and integration it is seen that $k_{m, p}-k_{p}$ equals, to the first order, the coefficient of $z_{p}$ in

i.e.

$$
\frac{Q_{m+1}(z)}{v(z)}
$$




$$
\left|k_{m, p}-k_{p}\right|=O\left(\left|v_{p}\right|\right)=O\left(\frac{1}{r_{0}^{p}}\right) .
$$

Because $r_{1}<1$ it is easily seen that (7.6), (7.7) and (7.8) also hold for $k_{n, \rightarrow p}$ and $k_{-p}$.

Whence by (6.11) we arrive at

$$
D_{m}\left(f_{m}\right)=\exp \left[\sum_{p=1}^{\infty} p k_{m, p} k_{-p}\right]=e^{\Lambda_{m}},
$$

which clearly converges by (7.6) and where

$$
f_{m}(z)=\frac{u\left(\frac{1}{z}\right)}{v_{m}(z)} .
$$

Taking the limit as $m \rightarrow \infty$ gives with aid of (7.8) the required result

$$
\lim _{m \rightarrow \infty} D_{m}\left(f_{m}\right)=\exp \left[\sum_{p=1}^{\infty} p k_{p} k_{-p}\right]=e^{\Lambda}
$$

which also converges by (7.6).

Alternatively we can employ the integral in (6.6) and show, using the uniformity of the convergence, that the double $\operatorname{limit}_{m \rightarrow \infty} \lim _{n \rightarrow \infty}$ may be taken under the integral sign, and that the series expansion of the integrand may be integrated term by term to give (7.13).

There remains to be shown that $\left[D_{m}\left(f_{m}\right)-D_{m}(f)\right] \rightarrow 0$ as $m \rightarrow \infty$, and for this use will be made of the Landsberg-Pólya representation which enables a Toeplitz determinant of order $n$ to be written as an $n$-fold multiple integral. The Toeplitz determinant $D_{n}(f)$ generated by the function $F(\theta)=f\left(e^{i \theta}\right)$, which has a Fourier series, may be written [13] as

$$
D_{n}(f)=\frac{2^{n(n-1)}}{n !} \cdot \frac{1}{(2 \pi)^{n}} \int_{0}^{2 \pi} \cdots \int_{0}^{2 \pi} \prod_{\mu<\nu}^{1 \cdots n} \sin ^{2}\left[\frac{\theta_{\mu}-\theta_{\nu}}{2}\right] \prod_{r=1}^{n} F\left(\theta_{r}\right) d \theta_{r} .
$$

On putting $F(\theta)=1$, i.e. $c_{0}=1$ and $c_{r}=c_{-r}=0$, this gives that

$$
1=\frac{2^{n(n-1)}}{n !} \cdot \frac{1}{(2 \pi)^{n}} \int_{0}^{2 \pi} \cdots \int_{0}^{2 \pi} \Pi^{\prime} \sin ^{2}\left[\frac{\theta_{\mu}-\theta_{\nu}}{2}\right] d \theta_{1} \cdots d \theta_{n},
$$

where $\Pi^{\prime}$ stands for the product ranging over $\mu, \nu=1, \cdots, n$ with $\mu<\nu$.

Hence it is clear that

$$
\left|D_{n}(f)\right| \leqq D_{n}(|f|) \leqq\left[\max _{\theta \in I_{1}}|F(\theta)|\right]^{n},
$$

where $I_{1}$ is the closed interval $[0,2 \pi]$. Since 


$$
\frac{2^{n(n-1)}}{n !} \sim\left(\frac{2^{n}}{n}\right)^{n} \rightarrow \infty
$$

this rules out any inequality using

$$
\frac{1}{2 \pi} \int_{0}^{2 \pi}|F(\theta)| d \theta
$$

mainly because no suitable upperbound, apart from unity, can be found for the product of sine terms. It is also obvious that if $|F(\theta)|<1$ then $D_{n}(f)$ decays exponentially to zero. The consequences of these results are that all the functions which will be dealt with in the remaining part of this paper will be assumed to satisfy either: (i) $|F(\theta)| \leqq r_{0}$ or (ii) $F(\theta)$ is real and positive?

Application of (7.14) to the function

and its rational approximant

$$
F(\theta)=f\left(e^{i \theta}\right)=\frac{u\left(e^{-i \theta}\right)}{v\left(e^{i \theta}\right)}
$$

$$
F_{m}(\theta)=\frac{u\left(e^{-i \theta}\right)}{v_{m}\left(e^{i \theta}\right)}
$$

both with $u_{0}=v_{0}=1$, and putting

$$
\text { and } \begin{aligned}
\zeta_{m} & =D_{m}\left(f_{m}\right)-D_{m}(f) \\
\zeta_{m, n} & =D_{m}\left(f_{m, n}\right)-D_{m}(f)
\end{aligned}
$$

gives that

$$
\begin{aligned}
\left|\zeta_{m}\right| & =\mid k_{m}^{\prime} \int_{0}^{2 \pi} \cdots \int_{0}^{2 \pi}\left[\prod_{r=1}^{m} F_{m}\left(\theta_{r}\right)-\prod_{r=1}^{m} F\left(\theta_{r}\right)\right] \\
& \times \Pi^{\prime} \sin ^{2}\left[\frac{\theta_{\mu}-\theta_{\nu}}{2}\right] d \theta_{1} \cdots d \theta_{m} \\
& \leqq \max _{\theta_{r} \in I_{1}}\left|\prod_{r=1}^{m} F_{m}\left(\theta_{r}\right)-\prod_{r=1}^{m} F\left(\theta_{r}\right)\right| \\
& \leqq M^{m-1} \max _{\theta_{r} \in I_{1}} \sum_{r=1}^{m}\left|F_{m}\left(\theta_{r}\right)-F\left(\theta_{r}\right)\right| \\
& \leqq m \cdot M^{m-1} \max _{\theta \in I_{1}}\left|F_{m}(\theta)-F(\theta)\right|
\end{aligned}
$$

where

$$
k_{m}^{\prime}=\frac{2^{m(m-1)}}{(2 \pi)^{m} m !} \text { and } M=\max _{\theta \in I_{1}}\left\{\left|F_{m}(\theta)\right|,|F(\theta)|\right\} .
$$

To obtain an estimate of (7.19) the following equality (omitting the argument $z$ ) will be used:

7 An alternative method is to use the fact that $c_{p}(m)=c_{p}+O\left(r_{0}^{-m}\right)$, and that the entries in $C^{-1}$ are bounded. This allows one to omit the last condition of theorem 2 . 


$$
\left|f_{m}-f\right|=\left|\frac{u}{v_{m}}-\frac{u}{v}\right|=|f|\left|\frac{v-v_{m}}{v_{m}}\right|=|f|\left|\frac{Q_{m+1}}{v_{m}}\right| .
$$

Now since

$$
\left|Q_{m+1}(z)\right|=O\left(\frac{1}{r_{0}^{m+1}}\right) \text { for } z \in \Omega
$$

and $F(\theta)$ is bounded, say $|F(\theta)| \leqq a$, then it is clear from (7.20) that

$$
\left|F_{m}(\theta)-F(\theta)\right| \leqq O\left(\frac{1}{r_{0}^{m+1}}\right) \text { and } M \leqq a\left[1+O\left(\frac{1}{r_{0}^{m+1}}\right)\right] .
$$

Substituting this in (7.19) gives that

$$
\left|\zeta_{m}\right| \leqq m \cdot a^{m-1}\left[1+O\left(\frac{1}{r_{0}^{m+1}}\right)\right]^{m-1} O\left(\frac{1}{r_{0}^{m+1}}\right),
$$

which tends to zero provided that $|F(\theta)| \leqq a<r_{0}\left(r_{0}>1\right)$.

Alternatively it may be written as

$$
\begin{aligned}
\left|\zeta_{m}\right| & \leqq \max _{\theta \in I_{1}}\left|\prod_{r=1}^{m} \frac{F_{m}\left(\theta_{r}\right)}{F\left(\theta_{r}\right)}-1\right| \cdot D_{m}(|f|) \\
& \leqq\left[\max _{\theta \in I_{1}}\left\{\left|\frac{F_{m}(\theta)}{F(\theta)}\right|, 1\right\}\right]^{m-1} \cdot m \cdot \max _{\theta \in I_{1}}\left|\frac{F_{m}(\theta)}{F(\theta)}-1\right| \cdot D_{m}(|f|)
\end{aligned}
$$

Since

$$
\left|\frac{F_{m}(\theta)}{F(\theta)}\right| \leqq 1+O\left(\frac{1}{r_{0}^{m+1}}\right)
$$

it follows that

$$
\left|\zeta_{m}\right| \leqq O\left(\frac{m}{r_{0}^{m+1}}\right)\left[1+O\left(\frac{1}{r_{0}^{m+1}}\right)\right]^{m-1} \cdot D_{m}(|f|),
$$

which tends to zero provided that $D_{m}(|f|)$ remains bounded or increases slower than $r_{0}^{m+1} / m$.

When the determinants are real it can be deduced that

$$
D_{m}(f)=D_{m}\left(f_{m}\right)\left[1 \pm O\left(\frac{m a^{m-1}}{r_{0}^{m+1}}\right)\right] .
$$

From (7.12), (7.13) and (7.8) we see that

$$
\left|e^{\Lambda_{m}}-e^{\Lambda}\right| \leqq O\left(\left|\Lambda_{m}-\Lambda\right|\right) \leqq O\left(\frac{1}{r_{0}^{2 m+2}}\right) .
$$

This combined with (7.22) shows that $D_{m}(f)$ tends to $e^{A}$ at a far slower rate than $D_{m}\left(f_{m}\right)$ does, and hence brings out the rather crude estimate of (7.22). 
In general when $u_{0} / v_{0} \neq 1$ it follows directly that

$$
\lim _{m \rightarrow \infty} D_{m}(f) \cdot e^{-m k_{0}}=e^{A} \text {. }
$$

\section{A generalization of Szegö's formula}

Using theorem $I$ the formula can now be extended to a restricted class of functions which have a Fourier series. The approach is similar to that used in the previous section except that now the region of overlap of convergence of the functions $u(1 / z)$ and $v(z)$ is reduced to just the unit circle.

More stringent conditions are necessary to ensure that

$$
\left[D_{m}\left(f_{m}\right)-D_{m}(f)\right] \cdot e^{-m k_{0}} \rightarrow 0
$$

and that

$$
\lim _{m \rightarrow \infty} D_{m}\left(f_{m}\right) \cdot e^{-m k_{0}}
$$

exists. Rather than assume the existence of the Fourier series of $\ln F(\theta)$, it will directly be assumed that $f\left(e^{i \theta}\right)$ is of the required structure as given in (7.1).

Definition. Let class $E$ be the class of all complex valued functions $f(z)$ satisfying the following conditions:

(a) $f(z)=\frac{u\left(\frac{1}{z}\right)}{v(z)}$,

(b) $u(z)=\sum_{p=0}^{\infty} u_{p} z^{p}(|z|<1), \quad v(z)=\sum_{p=0}^{\infty} v_{p} z^{p}(|z| \leqq 1)$,

(c) $u(z)$ and $v(z)$ are non-zero for $|z| \leqq 1$,

(d) $u^{\prime}\left(e^{i \theta}\right)$ rexists and $u^{\prime}\left(r e^{i \theta}\right) \rightarrow u^{\prime}\left(e^{i \theta}\right)$ point-wise as $r \rightarrow 1$,

(e) $\left|u^{\prime}\left(r e^{i \theta}\right)\right| \leqq K(\theta)$ for $0 \leqq r<1$, where $K(\theta) \in L^{2}(0,2 \pi)$,

(f) $m \cdot \sum_{p=m+1}^{\infty} v_{p} e^{i p \theta} \rightarrow 0$ uniformly in $\theta$ as $m \rightarrow \infty$,

$$
\text { (g) }\left|\frac{u\left(e^{-i \theta}\right) / u_{0}}{v\left(e^{i \theta}\right) / v_{0}}\right| \leqq 1
$$

THEOREM 3. If $f(z) \in E$, then the Toeplitz determinant $D_{m}(f)$ satisfies Szegö's formula

$$
\lim _{m \rightarrow \infty} D_{m}(f) \cdot e^{-m k_{0}}=\exp \left[\sum_{p=1}^{\infty} p k_{p} k_{-p}\right] .
$$


Proof. Without loss of generality we can assume that $u_{0}=v_{0}=1$.

If this is not the case we simply have to replace $D_{m}(f)$ by $D_{m}(f) \cdot e^{-m k_{0}}$ in the final result.

Conditions (b) and (c) imply that $\ln u(z)$ and $\ln v(z)$ are analytic for $|z|<1$ and hence can be expanded in power series as:

$$
\ln u(z)=\sum_{p=1}^{\infty} k_{-p} z^{p} \quad \text { and } \ln v(z)=-\sum_{p=1}^{\infty} k_{p} z^{p},
$$

where the principal branch of the logarithm is taken to ensure single valuedness. By assumption $(f)$ the partial sums $v_{m}(z)$ converge uniformly for $|z| \leqq 1$ to $v(z)$ and hence

$v(z)$ is uniformly continuous, bounded and non-zero for $|z| \leqq 1$. In particular, there exists $\delta>0$, such that $\left|v\left(e^{i \theta}\right)\right| \geqq \delta>0$ for all $\theta$.

Furthermore we have that $\ln v(z)$ is also uniformly continuous and bounded for $|z| \leqq 1$. This follows from the inequality

$$
\left|\ln z_{1}-\ln z_{2}\right| \leqq\left(\varepsilon+\frac{1}{\mu}\right)\left|z_{1}-z_{2}\right|
$$

where $z_{1}, z_{2} \neq 0,\left|z_{1}-z_{2}\right|<\delta_{1}$ and $\mu=\min \left\{\left|z_{1}\right|,\left|z_{2}\right|\right\}$. For sufficiently large $m$ the partial sums $v_{m}(z)$ are non-zero for $|z| \leqq 1$. For $|z|<1$ this follows immediately from Hurwitz's theorem [12], whereas for $|z|=1$ it can easily be deduced from (c) and (8.3).

Thus, for all $m>N_{1}$

$$
v_{m}\left(e^{i \theta}\right) \neq 0 .
$$

It will now be shown that the above three results also hold for $u(z)$ and its partial sums $u_{n}(z)$. From the existence of $u^{\prime}\left(e^{i \theta}\right)$ and the assumption (b) it follows that $u(z)$ and $\ln u(z)$ are uniformly continuous and hence bounded for $|z| \leqq 1$. Since both $u\left(e^{-i \theta}\right)$ and $1 / v\left(e^{i \theta}\right)$ are bounded it follows that the Fourier coefficients $c_{p}$ of

$$
F(\theta)=\frac{u\left(e^{-i \theta}\right)}{v\left(e^{i \theta}\right)}
$$

exist and are finite. In order to show that the partial sums $u_{n}\left(e^{i \theta}\right)$ are non-zero for sufficiently large $n$, we first have to prove the uniformity of their convergence. To do this we will make use of the following Lemmas.

Lemma 1. If $g(z)=\sum_{p=0}^{\infty} a_{p} z^{p}$ converges and is analytic for $|z|<1, g\left(e^{i \theta}\right)$ exists and $g\left(r e^{i \theta}\right) \rightarrow g\left(e^{i \theta}\right)$ pointwise as $r \rightarrow 1$, and $\left|g\left(r e^{i \theta}\right)\right| \leqq K(\theta)$, for $0 \leqq r<1$, where $K(\theta) \in L^{2}(0,2 \pi)$, then 

(i) $\sum_{p=0}^{\infty} a_{\nu} e^{i p \theta}$ is the Fourier series of $g\left(e^{i \theta}\right)$,
(ii) $g\left(e^{i \theta}\right) \in L^{2}(0,2 \pi)$,
(iii) $\frac{1}{2 \pi} \int_{0}^{2 \pi}\left|g\left(r e^{i \theta}\right)\right|^{2} d \theta=\sum_{p=0}^{\infty}\left|a_{p}\right|^{2} \gamma^{2 v}$ for $0 \leqq \gamma \leqq 1$.

Proof. (i) From the power series expansion it is clear that

$$
a_{\nu} r^{p}=\frac{1}{2 \pi} \int_{0}^{2 \pi} g\left(r e^{i \theta}\right) e^{-i p \theta} d \theta \text { for } 0 \leqq r<1 .
$$

Now $g\left(r e^{i \theta}\right) e^{-i p \theta}$ is dominated by $K(\theta) \in L^{2}(0,2 \pi)$, which by Schwarz's inequality is also in $L^{1}(0,2 \pi)$. Lebesgue's theorem on dominated convergence shows that it is permitted to take the limit as $r \rightarrow 1$ inside the integral, which indicates that $a_{p}$ is the Fourier coefficient of $g\left(e^{i \theta}\right)$, since $g\left(r e^{i \theta}\right) \rightarrow g\left(e^{i \theta}\right)$ pointwise as $r \rightarrow 1$.

(ii) For $|z|<1$ it follows directly on multiplication of $g(z)$ by its complex conjugate, and by the uniformity of the convergence, that (8.8) holds for $r<1$. Again applying the theorem on dominated convergence, but this time to $\left|g\left(r e^{i \theta}\right)\right|^{2}$, which is dominated by $K^{2}(\theta)$, gives that

(8.10) $\lim _{r \rightarrow 1} \frac{1}{2 \pi} \int_{0}^{2 \pi}\left|g\left(r e^{i \theta}\right)\right|^{2} d \theta=\lim _{r \rightarrow 1} \sum_{p=0}^{\infty}\left|a_{p}\right|^{2} \gamma^{2 p}=\frac{1}{2 \pi} \int_{0}^{2 \pi}\left|g\left(e^{i \theta}\right)\right|^{2} d \theta<\infty$,

which shows that $g\left(e^{i \theta}\right)$ is square integrable.

(iii) Using Fatou's Lemma it can be seen that

$$
\sum_{p=0}^{\infty}\left|a_{p}\right|^{2} \leqq \varliminf_{r \rightarrow 1} \sum_{p=0}^{\infty}\left|a_{p}\right|^{2} r^{2 \dot{p}}=\frac{1}{2 \pi} \int_{0}^{2 \pi}\left|g\left(e^{i \theta}\right)\right|^{2} d \theta<\infty,
$$

and hence by Abel's theorem it is clear that

$$
\lim _{r \rightarrow 1} \sum_{p=0}^{\infty}\left|a_{p}\right|^{2} \gamma^{2 p}=\sum_{p=0}^{\infty}\left|a_{p}\right|^{2}
$$

and so (8.8) also holds for $r=1$.

COROLLARY 1. The assumptions on $g(z)$ may be replaced by

(i) analytic for $|z|<1$

(ii) continuous for $|z| \leqq 1$

CoRollary 2. The results (8.6), (8.7) and (8.8) with $r=1$ also hold for $g\left(e^{-i \theta}\right)$. 
LEMMA 2. If

$$
g(z)=\sum_{p=0}^{\infty} a_{p} z^{p} \quad \text { and } \quad h(z)=\sum_{p=0}^{\infty} b_{-p} z^{p}
$$

satisfy the conditions of Lemma 1 , then

$$
\frac{1}{2 \pi} \int_{0}^{2 \pi} g\left(e^{i \theta}\right) h\left(e^{-i \theta}\right) d \theta=\sum_{p=0}^{\infty} a_{\nu} b_{-p} .
$$

Proof. By Lemma 1 we have that

$$
\begin{gathered}
g\left(e^{i \theta}\right)=\sum_{p=0}^{\infty} a_{p} e^{i p \theta} \\
h\left(e^{-i \theta}\right)=\sum_{p=0}^{\infty} b_{-p} e^{-i p \theta}
\end{gathered}
$$

and that both functions are in $L^{2}(0,2 \pi)$.

By Parseval's theorem the product of the Fourier series may be integrated term by term, giving (8.13).

Application of Lemma 1 to the function $u^{\prime}(z)$ shows that $u^{\prime}\left(e^{i \theta}\right)$ is square integrable and has Fourier series

with partial sums

$$
\sum_{p=1}^{\infty} p u_{p} e^{i(p-1) \theta}
$$

$$
u_{n}^{\prime}\left(e^{i \theta}\right)=\sum_{p=1}^{n} p u_{p} e^{i(p-1) \theta},
$$

and which may be integrated to give

$$
u\left(e^{i \theta}\right)=\sum_{p=0}^{\infty} u_{p} e^{i p \theta}
$$

Moreover $\sum_{p=0}^{\infty} p^{2}\left|u_{p}\right|^{2}$ converges and hence by Schwarz's inequality $\sum_{p=0}^{\infty}\left|u_{p}\right|$ also converges, implying that $u_{n}(z)$ converges uniformly to $u(z)$ for all $|z| \leqq 1$. Hence we deduce, as in (8.5), that $\exists N_{2}$, such that $u_{n}(z) \neq 0$ for all $n>N_{2}$ and $|z| \leqq 1$.

These results are obviously also true for $u_{n}\left(e^{-i \theta}\right)$. Let $N_{0}=\max \left\{N_{1}, N_{2}\right\}$, then for all $m, n>N_{0}$, we can form the rational approximant $u_{n}(1 / z) / v_{m}(z)$, and use theorem 1, which gives that

$$
D_{m}\left(f_{m, n}\right)=\exp \left[\frac{1}{2 \pi} \int_{0}^{2 \pi} \ln v_{m}\left(e^{i \theta}\right) \frac{u_{n}^{\prime}\left(e^{-i \theta}\right)}{u_{n}\left(e^{-i \theta}\right)}\left(-e^{-i \theta}\right) d \theta\right] .
$$

Under the assumptions made and with aid of the Lemmas we can now show that the double limit $\lim _{m \rightarrow \infty} \lim _{n \rightarrow \infty}$ may be taken under the integral sign 
in (8.18), and that it gives the required result. By the completeness theorem of Fourier series it follows that the partial sums in (8.16) converge in mean square to $u^{\prime}\left(e^{i \theta}\right)$. The same trivially holds for $u_{n}^{\prime}\left(e^{-i \theta}\right)$ and so

$$
\frac{1}{2 \pi} \int_{0}^{2 \pi}\left|u_{n}^{\prime}\left(e^{-i \theta}\right)-u^{\prime}\left(e^{-i \theta}\right)\right|^{2} d \theta \rightarrow 0 \text { as } n \rightarrow \infty .
$$

Similarly we have for $m>N_{0}$ that

$$
\frac{1}{2 \pi} \int_{0}^{2 \pi}\left|\ln v_{m}\left(e^{i \theta}\right)\left[\frac{1}{u_{n}\left(e^{-i \theta}\right)}-\frac{1}{u\left(e^{-i \theta}\right)}\right]\right|^{2} d \theta \rightarrow 0 \text { as } n \rightarrow \infty .
$$

Using the continuity of the inner product in the $L^{2}$ space ${ }^{8}$, and the fact that $u^{\prime}\left(e^{-i \theta}\right)$ and $\ln v_{m}\left(e^{i \theta}\right) / u\left(e^{-i \theta}\right)$ are in $L^{2}$, we obtain that as $n \rightarrow \infty$

$$
\frac{1}{2 \pi} \int_{0}^{2 \pi} \ln v_{m}\left(e^{i \theta}\right) \frac{d}{d \theta} \ln u_{n}\left(e^{-i \theta}\right) d \theta \rightarrow \frac{1}{2 \pi} \int_{0}^{2 \pi} \ln v_{m}\left(e^{i \theta}\right) \frac{d}{d \theta} \ln u\left(e^{-i \theta}\right) d \theta .
$$

It is a simple matter to verify from (8.3) and the assumptions $(d)$ and $(e)$, that for $|z|<1$ and $m>N_{0}$, the functions

$$
\ln v_{m}(z)=-\sum_{p=1}^{\infty} k_{m, p} z^{p} \text { and } \frac{u^{\prime}(z)}{u(z)}=\sum_{p=1}^{\infty} p k_{-p} z^{p}
$$

satisfy the conditions of Lemma 1 . Whence by Lemma 2 it immediately follows from (8.18) and (8.21) that

$$
\lim _{n \rightarrow \infty} D_{m}\left(f_{m, n}\right)=D_{m}\left(f_{m}\right)=\exp \left[\sum_{p=1}^{\infty} p k_{m, p} k_{-p}\right]<\infty .
$$

Since $u^{\prime}\left(e^{-i \theta}\right) / u\left(e^{-i \theta}\right)$ is in $L^{2}$ and $\ln v_{m}\left(e^{i \theta}\right)\left(m>N_{0}\right)$ converges boundedly (and in fact uniformly) and hence in mean square to $\ln v\left(e^{i \theta}\right)$, we can again apply the continuity of the inner product, (or the theorem on dominated convergence), which shows that the limit as $m \rightarrow \infty$ may be taken inside the integral (8.21) giving:

$$
\begin{aligned}
\lim _{m \rightarrow \infty} D_{m}\left(f_{m}\right) & =\lim _{m \rightarrow \infty} \frac{1}{2 \pi_{i}} \int_{0}^{2 \pi} \ln v_{m}\left(e^{i \theta}\right) \frac{d}{d \theta} \ln u\left(e^{-i \theta}\right) d \theta \\
& =\frac{1}{2 \pi_{i}} \int_{0}^{2 \pi} \ln v\left(e^{i \theta}\right) \frac{d}{d \theta} \ln u\left(e^{-i \theta}\right) d \theta .
\end{aligned}
$$

As in (8.22) we have that $\ln v(z)$ and $u^{\prime}(z) / u(z)$ satisfy the conditions of Lemma 1 and so by Lemma 2 we arrive at

$$
\lim _{m \rightarrow \infty} D_{m}\left(t_{m}\right)=\exp \left[\sum_{p=1}^{\infty} p k_{p} k_{-p}\right]<\infty .
$$

See Ref. 12, p. 390, ex. iv. 
It is noteworthy that Lemma 1 not only gives that $k_{-p}$ is the Fourier coefficient of $\ln u\left(e^{i \theta}\right)$ and that

$$
\left|k_{-p}\right|=o\left(\frac{1}{p}\right),
$$

but also that the series for $\ln u(z)$ in (8.2) converges for all $|z| \leqq 1$. We note that this cannot be deduced for the series for $\ln v(z)$, and hence the Fourier series $\sum_{-\infty}^{\infty} c_{p} e^{i p \theta}$ need not converge to $F(\theta)$.

There remains to be shown that the determinant $D_{m}\left(f_{m}\right)$ does in fact approximate $D_{m}(f)$ as $m \rightarrow \infty$. To do this we make use of (7.19), (7.20) and assumption $(g)$. Since $|F(\theta)| \leqq 1$ and $\left|v_{m}\left(e^{i \theta}\right)\right| \geqq \delta>0$ for all $m>N_{0}$, it is clear from (7.20) that

$$
m\left|F_{m}(\theta)-F(\theta)\right|=\left|\frac{F(\theta)}{v_{m}\left(e^{i \theta}\right)}\right| \cdot m\left|Q_{m+1}\left(e^{i \theta}\right)\right|,
$$

which by $(f)$ tends to zero uniformly in $\theta$. Furthermore we can deduce that for fixed $\theta$ either $\left|F_{m}(\theta)\right| \leqq|F(\theta)| \leqq 1$ or

$$
\left|F_{m}(\theta)\right| \leqq|F(\theta)|\left\{1+\left|\frac{Q_{m+1}\left(e^{i \theta}\right)}{v_{m}\left(e^{i \theta}\right)}\right|\right\},
$$

so that $M \leqq 1+o(1 / m)$. From (7.19) we now arrive at

$$
\left|\zeta_{m}\right| \leqq\left[1+o\left(\frac{1}{m}\right)\right]^{m-1} \cdot m \cdot \max _{\theta \in I_{1}}\left|F_{m}(\theta)-F(\theta)\right|,
$$

which by (8.27) tends to zero as $m \rightarrow \infty$.

Alternatively (7.23) can be used on exponentiating the product together with (8.4) and the facts that

$$
\left|e^{z}-1\right| \leqq|z| e^{|z|} \text { and } D_{m}(|f|) \leqq 1 .
$$

CoRollary. (i) Conditions (d), (e) and ( $f)$ may be replaced by the stronger but simpler conditions that on $[0,2 \pi]$

(1) $u^{\prime}\left(e^{i \theta}\right)$ exists and is continuous

(2) $v^{\prime}\left(e^{i \theta}\right)$ exists and satisfies a Lipschitz condition of order $\alpha$ with $0<\alpha<1$.

These conditions give rise to uniformity on the unit circle. The first assumption implies that $u(z)$ and $u^{\prime}(z)$ are uniformly continuous and bounded for all $|z| \leqq 1$, and hence $(d)$ and $(e)$ are satisfied. The second assumption, together with the fact that $v\left(e^{i \theta}\right)$ is non-zero, shows that (8.2) holds and also that 


$$
v\left(e^{i \theta}\right) \text { and } \frac{v^{\prime}\left(e^{i \theta}\right)}{v\left(e^{i \theta}\right)} \text { are in Lip } \alpha \quad(0<\alpha<1) .
$$

From Lemma 1 it can be seen that $u_{p}, v_{p}, p k_{-p}$ and $p k_{p}$ are the Fourier coefficients of their respective functions. This allows the application of two theorems (generalised to complex Fourier series) from the general theory of approximations [15], to the functions $v^{\prime}\left(e^{i \theta}\right) / v\left(e^{i \theta}\right)$ and $v\left(e^{i \theta}\right)$, giving that

$$
\left|k_{p}\right|=O\left(\frac{1}{p^{1+\alpha}}\right) \text { and }\left|Q_{p+1}\left(e^{i \theta}\right)\right|=O\left(\frac{\ln p}{p^{1+\alpha}}\right) \quad(p>0) .
$$

Whence assumption $(f)$ is now also satisfied. Moreover it follows that (8.2) holds for all $|z| \leqq 1$ and so

$$
\ln F(\theta)=\sum_{-\infty}^{\infty} k_{p} e^{i p \theta} \text { with } \frac{u_{0}}{v_{0}}=e^{k_{0}},
$$

and furthermore $F(\theta)=\sum_{-\infty}^{\infty} c_{p} e^{i p \theta}$.

(ii) We note that the existence and finiteness of $v^{\prime}\left(e^{i \theta}\right)$ does not necessarily imply that $m Q_{m+1}\left(e^{i \theta}\right) \rightarrow 0$ or vice versa.

\section{The Hermitian case}

Suppose that $F(\theta) \in L^{1}(0,2 \pi)$ and that $F(\theta)$ is contained in class $A$, where class $A$ is the class of all functions satisfying:

(1) $F(\theta)$ is real and positive

(2) $F(\theta)$ is periodic with period $2 \pi$

(3) $F^{\prime}(\theta)$ exists for all $\theta$ and is in $\operatorname{Lip} \alpha$ with $0<\alpha<1$ [3].

It will now be shown that under these conditions theorem 3 may be applied. This is possible since now the functions $u\left(e^{-i \theta}\right)$ and $v\left(e^{i \theta}\right)$ are closely related such that the representation of $F(\theta)$ in the form $u\left(e^{-i \theta}\right) / v\left(e^{i \theta}\right)$ reduces to the positive function representation $\left|h\left(e^{i \theta}\right)\right|^{2}$. Since $F(\theta)$ is positive and periodic we have that

(a) $\ln F(\theta)$ is real and periodic

(b) $\ln F(\theta) \leqq F(\theta)$

(c) $D_{m}(|F|)=D_{m}(F)>0$

(d) $F(\theta)$ and $\ln F(\theta)$ are bounded and have Fourier series

$\sum_{-\infty}^{\infty} c p e^{i p \theta}$ and $\sum_{-\infty}^{\infty} k_{p} e^{i p \theta}$ with $c_{p}=\bar{c}_{-p}, k_{p}=k_{-p}$ and $k_{0}$ real respectively. Condition (3) implies, since $F(\theta)$ is bounded and positive, that 


$$
F(\theta) \text { and } \frac{F^{\prime}(\theta)}{F(\theta)} \text { are both in } \operatorname{Lip} \alpha \quad(0<\alpha<1) .
$$

Application of (8.33) shows that

$$
c_{p} \text { and } k_{p} \text { are of order } O\left(\frac{1}{p^{1+a}}\right) \text {. }
$$

Hence the Fourier series in (9.2) converge uniformly to their continuous limit functions. As before $F(\theta)$ can be written in the form $u\left(e^{-i \theta}\right) / v\left(e^{i \theta}\right)$ by defining

$$
u\left(\frac{1}{z}\right) / u_{0}=\exp \left[\sum_{p=1}^{\infty} k_{-\triangleright} z^{p}\right]=u_{0}+\frac{u_{1}}{z}+\cdots+\frac{u_{n}}{z^{n}}+\cdots \quad(|z| \geqq 1),
$$

$$
\begin{aligned}
& v(z) / v_{0}=\exp \left[-\sum_{p=1}^{\infty} k_{p} z^{p}\right]=v_{0}+v_{1} z+\cdots+v_{n} z^{n}+\cdots \quad(|z| \leqq 1) \\
& \text { and }
\end{aligned}
$$

$$
\frac{u_{0}}{v_{0}}=e^{k_{0}}>0
$$

$$
\left(u_{0} \neq 0 \neq v_{0}\right)
$$

From the fact that $k_{p}=k_{-p}$, we obtain that

and hence

$$
\overline{u\left(e^{-i \theta}\right) / u_{0}}=\frac{1}{v\left(e^{i \theta}\right) / v_{0}}
$$

$$
F(\theta)=|F(\theta)|=\frac{u\left(e^{-i \theta}\right)}{v\left(e^{i \theta}\right)}=\frac{u_{0}}{v_{0}} \cdot\left|\frac{1}{v\left(e^{i \theta}\right) / v_{0}}\right|^{2}>0 .
$$

Thus the two representations are equivalent if $h(z)=v_{0} / v(z)$. Using the notation of [3], the function $k(z)$ is defined by

$$
\frac{1}{2} k(z)=-\ln \left(v(z) / v_{0}\right)=\sum_{p=1}^{\infty} k_{p} z^{p} \quad(|z| \leqq 1),
$$

from which it is seen that $v(z) \neq 0$ for $|z| \leqq 1$ and that

$$
\ln F(\theta)=k_{0}-2 \operatorname{Re}\left[\ln \left(v\left(e^{i \theta}\right) / v_{0}\right)\right]=k_{0}+\operatorname{Re}\left[k\left(e^{i \theta}\right)\right] .
$$

Consequently $k(z)$ and $v(z) / v_{0}=\exp \left[-\frac{1}{2} k(z)\right]$ are continuous for $|z| \leqq 1$. It was shown in [3] that the functions $k^{\prime}(z), v^{\prime}(z) / v(z)$ and $v^{\prime}(z) / v_{0}$ are all continuous for $|z| \leqq 1$ and satisfy the same Lipschitz condition on $C_{0}$. Whence in view of the general theorems on approximation, it follows as in (8.33) that

$$
\left|Q_{m+1}\left(e^{i \theta}\right)\right|=O\left(\frac{\ln m}{m^{1+\alpha}}\right) .
$$


Direct application of theorem 3 Cor. (i) shows that

$$
\left|\zeta_{m}\right| \leqq 0\left(\frac{\ln m}{m^{\alpha}}\right) \cdot D_{m}(F) .
$$

It is clear that the condition $(g)$ of theorem 3 may be removed in this case since $F(\theta)$ is positive. Thus

$$
\left|\frac{D_{m}\left(F_{m}\right)}{D_{m}(F)}-1\right| \leqq O\left(\frac{\ln m}{m^{\alpha}}\right) \quad(0<\alpha<1),
$$

which tends to zero as $m$ tends to infinity, and hence

$$
D_{m}(F) \cdot e^{-m k_{0}} \rightarrow \exp \left[\sum_{p=1}^{\infty} p\left|k_{p}\right|^{2}\right]
$$

which converges by (9.4) to a positive limit. It was shown by Szegö that in addition the sequences $\left\{D_{m-1}(F) / D_{m}(F)\right\}$ and $\left\{D_{m}(F)\right\}$ are positive and increasing, the former being bounded above by $e^{-k_{0}}$. The corresponding sequences with $F$ replaced by $F_{m, n}$ or $F_{m}$, do not have these properties since $k_{m, y}$ need not equal $k_{m,-s}$ for $p>m$, i.e. the rational approximant $F_{m, m}$ is not real in general.

\section{Unimodular generating functions}

In the special case where $|F(\theta)|=1$, the Theorems 1,2 and 3 can be simplified somewhat since now $F(\theta)$ may be represented as $e^{i \delta(\theta)}$, where $\delta(\theta)$ is real valued. If $\ln F(\theta)=\sum_{-\infty}^{\infty} k_{p} e^{i p \theta}=i \delta(\theta)$ converges for all $\theta$, and $k_{0}=0$, then $k_{p}=-k_{-p}$, both of which are real, and further $\delta(\theta)=2 \sum_{p=1}^{\infty} k_{p} \sin p \theta$ is an odd function of $\theta$. Defining

$$
v(z)=\exp \left[-\sum_{p=1}^{\infty} k_{p} z^{p}\right]=1+v_{1} z+\cdots+v_{n} z^{n}+\cdots
$$

which converges and is non-zero for $|z| \leqq 1$, shows that

$$
F(\theta)=f\left(e^{i \theta}\right)=\frac{v\left(e^{-i \theta}\right)}{v\left(e^{i \theta}\right)} .
$$

Alternatively it may be assumed that $f(z)$ has this form, with $v(z)=1+v_{1} z+\cdots$ being convergent and non-zero for $|z| \leqq r_{0}\left(r_{0}>1\right)$, e.g. $v(z)=(1-A z)^{\frac{1}{2}}$, with $A<1$. On defining

$$
\ln v_{m}(z)=-\sum_{p=1}^{\infty} k_{m, p} z^{p} \quad\left(|z| \leqq r_{0}\right)
$$

with $k_{m, p}=k_{p}$ for $p \leqq m$, the rational approximant 


$$
F_{m, m}(\theta)=\frac{v_{m}\left(e^{-i \theta}\right)}{v_{m}\left(e^{i \theta}\right)}
$$

can be written as $e^{i \delta_{m}(\theta)}$, where $\delta_{m}(\theta)=2 \sum_{p=1}^{\infty} k_{m, y} \sin p \theta$, which is an odd function of $\theta$ and which is real if and only if the coefficients $v_{p}$ are real. From (7.14) it follows that

(10.2) $\zeta_{m, m}=k_{m}^{\prime} \int_{0}^{2 \pi} \cdots \int_{0}^{2 \pi} e^{i \Delta}\left[e^{i\left(\Delta_{m}-\Delta\right)}-1\right] \Pi^{\prime} \sin ^{2}\left(\frac{\theta_{\mu}-\theta_{\nu}}{2}\right) d \theta_{1} \cdots d \theta_{m}$,

where $\Delta=\delta\left(\theta_{1}\right)+\cdots+\delta\left(\theta_{m}\right), \Delta_{m}=\delta_{m}\left(\theta_{1}\right)+\cdots+\delta_{m}\left(\theta_{m}\right)$ and

$$
\Delta_{m}-\Delta=2 \sum_{p=m+1}^{\infty}\left(k_{m, p}-k_{p}\right)\left(\sin p \theta_{1}+\cdots+\sin p \theta_{m}\right) \text {. }
$$

The exponential can now be expanded as

$$
e^{i\left(\Delta_{m}-\Delta\right)}-1=i\left(\Delta_{m}-\Delta\right)-\frac{1}{2}\left(\Delta_{m}-\Delta\right)^{2}+\cdots
$$

in which the first term may be taken as the principal term provided that $\left|k_{m, p}-k_{p}\right|=O\left(1 / r_{0}^{p+1}\right)$. If this is the case then termwise integration is permitted showing that

$$
\left|\zeta_{m, m}\right|=O\left(m\left|k_{m, m+1}-k_{m+1}\right|\right)=O\left(\frac{m}{r_{0}^{m+1}}\right) .
$$

The same result is obtained on using $F_{m}(\theta)=\left(v\left(e^{-i \theta}\right) / v_{m}\left(e^{i \theta}\right)\right)$ and $\zeta_{m}$. The second term in the expansion is of order $O\left(\mathrm{~m}^{2} / \mathrm{r}_{0}^{2 m+2}\right)$ and is much closer to the expected rate of (7.26). To obtain this it would thus be necessary to show that the first term in (10.4) vanishes after integration, i.e.

$$
\begin{aligned}
& i \sum_{p=m+1}^{\infty}\left(k_{m, p}-k_{p}\right) \\
& \times k_{m}^{\prime} \int_{0}^{2 \pi} \cdots \int_{0}^{2 \pi} e^{i \Delta}\left(\sin p \theta_{1}+\cdots+\sin p \theta_{m}\right) \Pi^{\prime} \sin ^{2}\left(\frac{\theta_{\mu}-\theta_{\nu}}{2}\right) d \theta_{1} \cdots d \theta_{n}
\end{aligned}
$$

must vanish. Since $\delta(\theta)$ is an odd function of $\theta$ and the product of sine terms is even, it follows that (10.6) reduces to the sum of integrals of the form

$$
\int_{0}^{2 \pi} \cdots \int_{0}^{2 \pi} \sin \Delta \cdot \sin p \theta_{1} \Pi^{\prime} \sin ^{2}\left(\frac{\theta_{\mu}-\theta_{\nu}}{2}\right) d \theta_{1} \cdots d \theta_{m} .
$$

Unfortunately, this integral is rather intractable, even in very special cases, and hence brings out clearly the shortcomings of the LandsbergPólya representation. 


\section{Non uniform approximations}

Because of the large number of applications of Szegö's formula, it would be desirable to extend it to the non-uniform case in which the generating function $f(z)=(u(1 / z) / v(z))$ is not approximated uniformly on the unit circle $C_{0}$ by its rational approximant $f_{m}(z)=\left(u(1 / z) / v_{m}(z)\right)$, which satisfies the conditions of theorem 1 . One would expect that if $F(\theta)=f\left(e^{i \theta}\right)$ is in $L^{1}(0,2 \pi)$, then a discontinuity on $C_{0}$, causing the nonuniformity of convergence, would not affect the individual integrals in the Landsberg-Pólya representation. However it appears not possible to extend it since the range of integration in (7.14) would have to be split up in order to isolate the behaviour near the discontinuity.

Suppose for example that

$$
F(\theta)=\left(\frac{1-e^{-i \theta}}{1-e^{i \theta}}\right)^{\alpha}=e^{i \alpha(\pi-\theta)} \quad(0<\alpha<1),
$$

then

$$
|F(\theta)|=1, v(z)=(1-z)^{\alpha}=\sum_{t=0}^{\infty} \frac{(-\alpha) t}{t !} z^{t}
$$

and

$$
\left|v_{m}(z)\right| \geqq v_{m}(1)=\frac{(1-\alpha)_{m}}{m !}>0,
$$

Using Abel's method of summation we obtain that

$$
\left|Q_{m+1}\left(e^{i \theta}\right)\right|=\left|v\left(e^{i \theta}\right)-v_{m}\left(e^{i \theta}\right)\right| \leqq O\left[\frac{1}{(m \varepsilon)^{1+\alpha}}\right] \quad(\varepsilon<\theta<2 \pi-\varepsilon),
$$

and hence by $(7.20)$

$$
\left|F_{m}(\theta)-F(\theta)\right| \leqq \begin{cases}O\left[\frac{1}{(m \varepsilon)^{1+\alpha}}\right] & (\varepsilon<\theta<2 \pi-\varepsilon) \\ 1 & (\text { all } \theta),\end{cases}
$$

since

$$
\left|\frac{Q_{m+1}\left(e^{i \theta}\right)}{v_{m}\left(e^{i \theta}\right)}\right| \leqq 1
$$

for all $\theta$. To cope with this non uniformity we have to consider for every dummy variable $\theta_{r}$ in (7.18) the intervals $(0, \varepsilon),(\varepsilon, 2 \pi-\varepsilon)$ and $(2 \pi-\varepsilon, 2 \pi)$, where $\varepsilon$ is any positive number, giving altogether $3^{m}$ integrals. The principal contribution $J$ comes from the integral with the range $(\varepsilon, 2 \pi-\varepsilon) m$-fold repeated. Taking moduli gives that

$$
|J| \leqq O\left[\frac{m}{(m \varepsilon)^{1+\alpha}}\right] \cdot\left[1-\frac{(2 \varepsilon)^{n(n-1)}}{(2 \pi)^{n} n !}\right],
$$


which tends to zero for suitable $\varepsilon=\varepsilon(n)$, say $\varepsilon=n^{-\delta}$ with $\delta<\alpha /(1+\alpha)$. Using (7.15), (11.4) and the fact that the product of sine terms is a positive function in each of the variables $\theta_{r}$, it is easily shown that the sum of the remaining $3^{m}-1$ integrals of (7.18), will tend to zero if $\varepsilon=O\left(12^{-n}\right)$, which is far too small for (11.5) to be possible.

\section{Conclusions}

In this paper we have shown how the resultant approach, consisting of

(i) the theory of the resultant itself, and

(ii) the study of the approximating ability of the resultant,

can be used to evaluate the limit of certain Toeplitz determinants. The approach is satisfactory in so far that it gives us in (i) a powerful tool which not only permits an exact evaluation, but also gives an asymptotic expansion from which certain correct limits can be calculated. The theory of resultant has not been investigated a great deal in this particular context and its applications have by no means been exhausted. In the study of the approximations of a Toeplitz determinant it is an essential requirement that it must be shown that the approximation taken does in fact approximate the original determinent, a requirement that only too often conveniently is neglected, and it is here that the shortcomings of the second part (ii) of the approach become evident, since no tractable representation has yet been developed. We have seen that the Landsberg-Pólya representation of a Toeplitz determinant can be used to derive some useful results such as the exact limit of the determinants, however due to analytical difficulties associated with the multiple integral it neither gives an adequate insight into the exact rate at which the determinant tends to its limit, nor can it handle the non uniform approximations of the generating function. It is clear that to overcome these obstacles either a far more refined approach using the Landsberg-Pólya representation has to be developed, or an alternative more powerful representation has to be derived.

In a later publication it is hoped to show how the resultant approach can be applied to the Toeplitz determinants representing the Ising model spin-spin correlations, and how the results agree with numerical and physical evidence obtained so far.

\section{References}

[1] O. Toeplitz, 'Theorie der L-Formen', Math. Ann. 70 (1910), 351-376.

[2] U. Grenander and G. Szegö, 'Toeplitz forms and their applications' (University of California Press, and Cambridge University Press, Cambridge, England, 1950).

[3] G. Szegö, 'On certain Hermitian forms associated with the Fourier series of a positive function', Communications du séminaire mathématique de l'université de Lund, tome supplémentaire (1952), 228-238. 
[4] B. L. van der Waerden, 'Modern algebra', (Frederick Ungar Publishing Co., New York, 1948, vol. 1, p. 83).

[5 ]H. S. Green, 'The long-range correlations of various Ising lattices', Z. Phys. 171 (1963), 129-148.

[6] B. Kaufman and L. Onsager, 'Long-range order' (unpublished).

[7] L. P. Kadanoff, 'Spin-Spin Correlation in the Two Dimensional Ising Model', Il Nuovo Cimento 44 (1966), $276-305$.

[8] R. W. Gibberd and C. A. Hurst, 'A new approach to the Ising model', J. Math. Phys. 8 (1967), $1427-1435$.

[9] F. R. Gantmacher, Theory of matrices (Chelsea, New York, 1959, vol. 2, Sec. 7, p. 196) (see also revised edition).

[10] T. Muir and W. H. Metzler, Treatise on the theory of determinants (Albany, New York, 1930 (privately published), Secs. 81 and 465).

[11] E. C. Titchmarsh, The theory of functions, (Oxford, 1939, second edition, Sec. 3.45, p. 119).

[12] G. Szegö, 'Ein Grenzwertsatz über die Toeplitzschen Determinanten einer reellen positiven Funktion', Math. Ann. 76 (1915), 490-603.

[13] C. de la Vallée Poussin, Leçons sur l'Approximation des functions d'une variable véelle (Gauthier-Villards, Paris, 1919, Secs. 12, 21).

[14] E. W. Montroll, R. B. Potts and. J. C. Ward, 'Correlations and Spontaneous Magnetization of the Two-Dimensional Ising Model', $J$. of Math. Phys. 4 (1963), 308-322.

[15] M. Kac, 'Toeplitz matrices, translation kernels, and a related problem in probability theory', Duke Math. J., 21 (1954), 501-509.

[16] M. Kac, Probability and Related Topics in Physical Sciences (Interscience Publishers, New York, 1959), 40-57.

[17] G. Baxter, 'Polynomials defined by a difference system', J. Math. Anal. and Appl., 2 (1961), $223-263$.

[18] G. Baxter, 'A convergence equivalence related to polynomials orthogonal on the unit circle', Trans. Amer. Math. Soc., 99 (1961), $471-487$.

[19] G. Baxter, 'A norm inequality for a 'finite section' Wiener-Hopf equation', Illinois $J$. of Math., 7 (1963), 97-103.

[20] I. I. Hirschman, Jr., 'The strong Szegö limit theorem for Toeplitz determinants', Amer. J. Math., 88 (1966), 577-614.

[21] A. Devinatz, 'The strong Szegö limit theorem', Illinois J. of Math., 11 (1967), $160-175$.

[22] I. I. Hirschman, Jr., 'Recent developments in the theory of finite Toeplitz operators', to be published.

[23] G. V. Ryazanov, 'Correlation asymptotics for a plane Ising lattice', Sov. Phys. $J E T P, 22(1966), 789-795$.

[24] T. T. Wu, 'Theory of Toeplitz determinants and spin correlations of the two-dimensional Ising model I', Phys. Rev., 149 (1966), 380-401.

[25] B. M. McCoy and T. T. Wu, 'Theory of Toeplitz determinants and spin correlations of the two-dimensional Ising model II', Phys. Rev., 155 (1967), 438-452.

[26] $\mathrm{H}$. Cheng and T. T. Wu, 'Theory of Toeplitz determinants and spin correlations of the two-dimensional Ising model III', Phys. Rev., 164 (1967), $719-735$.

[27] B. M. McCoy and T. T. Wu, 'Theory of Toeplitz determinants and spin correlations of the two-dimensional Ising model IV', Phys. Rev., 162 (1967), 436-475.

The University of Adelaide

Adelaide 